\title{
Article
}

\section{Earth-Moon Lagrangian points as a testbed for general relativity and effective field theories of gravity}

Battista, Emmanuele, Dell'Agnello, Simone, Di Fiore, Luciano, Esposito, Giampiero, Simo, Jules and Grado, Aniello

Available at https://clok.uclan.ac.uk/13395/

Battista, Emmanuele, Dell'Agnello, Simone, Di Fiore, Luciano, Esposito, Giampiero, Simo, Jules orcid iconORCID: 0000-0002-1489-5920 and Grado, Aniello (2015) Earth-Moon Lagrangian points as a testbed for general relativity and effective field theories of gravity. Physical Review D (PRD), 92 (6). 064045. ISSN 1550-7998

It is advisable to refer to the publisher's version if you intend to cite from the work. http://dx.doi.org/10.1103/PhysRevD.92.064045

For more information about UCLan's research in this area go to http://www.uclan.ac.uk/researchgroups/ and search for <name of research Group>.

For information about Research generally at UCLan please go to http://www.uclan.ac.uk/research/

All outputs in CLoK are protected by Intellectual Property Rights law, including Copyright law. Copyright, IPR and Moral Rights for the works on this site are retained by the individual authors and/or other copyright owners. Terms and conditions for use of this material are defined in the policies page.

\section{CLoK}

Central Lancashire online Knowledge www.clok.uclan.ac.uk

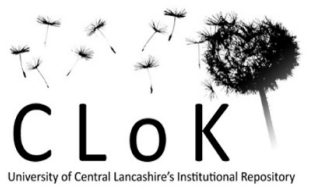




\title{
Earth-Moon Lagrangian points as a testbed for general relativity and effective field theories of gravity
}

\author{
Emmanuele Battista* \\ Dipartimento di Fisica, Complesso Universitario di Monte S. Angelo, \\ Via Cintia Edificio 6, 80126 Napoli, Italy \\ Istituto Nazionale di Fisica Nucleare, Sezione di Napoli, \\ Complesso Universitario di Monte S. Angelo, \\ Via Cintia Edificio 6, 80126 Napoli, Italy \\ Simone Dell'Agnello ${ }^{\dagger}$ \\ Istituto Nazionale di Fisica Nucleare, \\ Laboratori Nazionali di Frascati, 00044 Frascati, Italy \\ Giampiero Esposito ${ }^{\ddagger}$ and Luciano Di Fiore ${ }^{\S}$ \\ Istituto Nazionale di Fisica Nucleare, Sezione di Napoli, \\ Complesso Universitario di Monte S. Angelo, \\ Via Cintia Edificio 6, 80126 Napoli, Italy \\ Jules Simo** \\ Department of Mechanical and Aerospace Engineering, \\ University of Strathclyde, Glasgow, G1 1XJ, United Kingdom \\ School of Engineering, University of Central Lancashire, \\ Preston, PR1 2HE, United Kingdom \\ Aniello Grado ${ }^{\dagger \dagger}$ \\ INAF, Osservatorio Astronomico di Capodimonte, 80131 Napoli, Italy
}

(Dated: September 9, 2015) 


\begin{abstract}
We first analyse the restricted four-body problem consisting of the Earth, the Moon and the Sun as the primaries and a spacecraft as the planetoid. This scheme allows us to take into account the solar perturbation in the description of the motion of a spacecraft in the vicinity of the stable Earth-Moon libration points $L_{4}$ and $L_{5}$ both in the classical regime and in the context of effective field theories of gravity. A vehicle initially placed at $L_{4}$ or $L_{5}$ will not remain near the respective points. In particular, in the classical case the vehicle moves on a trajectory about the libration points for at least 700 days before escaping away. We show that this is true also if the modified long-distance Newtonian potential of effective gravity is employed. We also evaluate the impulse required to cancel out the perturbing force due to the Sun in order to force the spacecraft to stay precisely at $L_{4}$ or $L_{5}$. It turns out that this value is slightly modified with respect to the corresponding Newtonian one. In the second part of the paper, we first evaluate the location of all Lagrangian points in the Earth-Moon system within the framework of general relativity. For the points $L_{4}$ and $L_{5}$, the corrections of coordinates are of order a few millimeters and describe a tiny departure from the equilateral triangle. After that, we set up a scheme where the theory which is quantum corrected has as its classical counterpart the Einstein theory, instead of the Newtonian one. In other words, we deal with a theory involving quantum corrections to Einstein gravity, rather than to Newtonian gravity. By virtue of the effective-gravity correction to the longdistance form of the potential among two point masses, all terms involving the ratio between the gravitational radius of the primary and its separation from the planetoid get modified. Within this framework, for the Lagrangian points of stable equilibrium, we find quantum corrections of order two millimeters, whereas for Lagrangian points of unstable equilibrium we find quantum corrections below a millimeter. In the latter case, for the point $L_{1}$, general relativity corrects Newtonian theory by 7.61 meters, comparable, as an order of magnitude, with the lunar geodesic precession of about 3 meters per orbit. The latter is a cumulative effect accurately measured at the centimeter level through the lunar laser ranging positioning technique. Thus, it is possible to study a new laser ranging test of general relativity to measure the 7.61-meter correction to the $L_{1}$ Lagrangian point, an observable never used before in the Sun-Earth-Moon system. Performing such an experiment requires controlling the propulsion to precisely reach $L_{1}$, an instrumental accuracy comparable to the measurement of the lunar geodesic precession, understanding systematic effects resulting from thermal radiation and multi-body gravitational perturbations. This will then be the basis to
\end{abstract}


consider a second-generation experiment to study deviations of effective field theories of gravity from general relativity in the Sun-Earth-Moon system.

PACS numbers: 04.60.Ds, 95.10.Ce

*E-mail: ebattista@na.infn.it

$\dagger$ E-mail: simone.dellagnello@lnf.infn.it

${ }^{\ddagger}$ E-mail: gesposit@na.infn.it

$\S$ E-mail: luciano.difiore@na.infn.it

${ }^{* *}$ E-mail: jules.simo@strath.ac.uk

${ }^{\dagger \dagger}$ E-mail: aniello.grado@gmail.com 


\section{INTRODUCTION}

In the space surrounding two bodies that orbit about their mutual mass center there are five points where a third body will remain in equilibrium under the gravitational attraction of the other two bodies. These points are called Lagrangian points in honour of Joseph Lagrange, who discovered them in 1772 while studying the restricted problem formed by the Sun-Jupiter system. The discovery of their physical realization, i.e. the Trojan group of asteroids, began only in 1906 thanks to the astronomer Max Wolf with the first-seen member of this group, 588 Achilles, which is located near the triangular libration point of the Sun-Jupiter system. Today we know that there are 3898 known Trojans at the triangular Lagrangian point $L_{4}$ and 2049 at $L_{5}$ [1]. In the sixties, simultaneously with the increased interest in space explorations, the question of existence of Lagrangian points with respect to other primaries, especially for the Earth-Moon system, arose quite naturally. In fact, if there are stable stationary solutions for various primary combinations, then from a practical point of view placing observational platforms at these points becomes feasible, especially in a really close and accessible system like the Earth-Moon system, which is also the most convenient system from an economic point of view. While the Sun-Jupiter system clearly possesses a collection of asteroids at the triangular libration points, the ability of the Earth-Moon system to collect debris or dust at the corresponding points and in what is called Kordylewski clouds is still in question (see Ref. [2] for further details). The major perturbing effect on the Trojans is represented by Saturn, while the stabilizing forces come from the Sun and Jupiter. The major perturbation on the Earth-Moon libration clouds is the Sun and the stabilizing effects are derived from the Earth and the Moon. This explains why the existence of accumulated material at $L_{4}$ or $L_{5}$ in the Earth-Moon system is not so obvious. Bodies at the triangular libration points of the system consisting of the Sun and another planet would face the perturbations from Jupiter; therefore, it is not surprising that the only currently known material accumulation is confined to the Sun-Jupiter system, although some asteroids were found also in the Sun-Earth system around the libration point $L_{4}$, as is shown by recent observations [3]. As far as the collinear Lagrangian points for the Earth-Moon system are concerned, we know that $L_{1}$ allows comparatively easy access to Lunar and Earth orbits with minimal change in velocity and has this as an advantage to position a half-way manned space station intended to help transport cargo and personnel 
to the Moon and backwards, whereas $L_{2}$ would be a good location for a communications satellite covering the Moon's far side and would be an ideal location for a propellant depot as part of the proposed depot-based space transportation architecture [4].

Recently, inspired by the works in Refs. [5-12] on effective field theories of gravity, some of us [13-15] have applied this theoretical analysis to the macroscopic bodies occurring in celestial mechanics [16-18], especially in the Earth-Moon system. It has been demonstrated that in the quantum regime, when only the interaction potential is modified in the Lagrangian of Newtonian gravity, the position of collinear Lagrangian points is governed by four algebraic ninth degree equations, which reduce to two algebraic fifth degree equations in the classical regime, while the quantum corrected position of the noncollinear libration points is described in terms of a pair of quintic equations, which predict that the classical equilateral triangle picture is no longer valid in the quantum scheme. For the Earth-Moon system, the prediction about the discrepancy between classical and quantum corrected quantities is of the order of millimeters. This magnitude is comparable with the instrumental accuracy of point-to-point laser Time-of-Flight ( ToF) measurements in space typical of the modern Satellite/Lunar Laser Ranging (SLR/LLR) techniques [19-32]. The full positioning error budget of the orbits of satellites equipped with laser retroreflectors and reconstructed by laser ranging depends also on other sources of uncertainty (related to the specific orbit, satellite and retroreflector array), in addition to the pure point-to-point laser ToF instrumental accuracy (related to the network of laser ranging ground stations of the ILRS, i.e., the International Laser Ranging Service [20]. The full positioning error budget can be larger than millimeters.

This is an interesting potentiality, because we are dealing with predictions which might become testable in the Earth-Moon system. This is a novel feature in the theory of quantum gravity, because all other theories are so far unable to produce testable effects [33-43]. These predictions become more realistic if we include the perturbations due to the gravitational presence of the Sun, in other words we have to face up the restricted problem of four bodies, consisting of the Sun, the Earth and the Moon as the three primaries and the fourth body (e.g. the laser-ranged test mass, a spacecraft or by exploiting the solar sail technology $[15,44-51])$ which has an infinitesimal mass, to avoid affecting the motion of the primaries. As we know, in the restricted three-body problem the motion of the two primaries is exactly described by the equations of motion governing the two-body problem. Therefore, we may 
generalize the problem first by solving the dynamical equations describing the motion of the three primaries and then by finding the motion of the planetoid in the presumably known gravitational field produced by the primaries. Since no closed-form solution is known for the full three-body problem, this generalization to the case of four bodies is rather difficult. A practicable possibility consists in assuming the motions of the three primaries and, without attempting to establish the exact solution of the equations governing these motions, accept an approximate solution. Such an approximation may be, for instance, that the Earth and the Moon move in elliptic orbits around their mass center and that the mass center of the Earth-Moon system, in turn, moves in elliptic orbit around the Sun. The plane of the orbit of the mass center of the Earth-Moon system, which is called the plane of ecliptic, is inclined relative to the plane containing the orbits of the Earth and the Moon. A simpler approximation would consist in neglecting the eccentricity of all orbits, i.e. assuming that the Earth, the Moon and their mass center have circular orbits. Under these assumptions, the authors of Ref. [52] did show that, although it is widely accepted that, with the introduction of the Sun, the points $L_{4}$ and $L_{5}$ of the Earth-Moon system cease to be equilibrium points, stable motion may be possible in a region around these noncollinear libration points. The term "stable" here indicates that the planetoid will remain within a certain region for the period of time during which the motion is studied. The work in Ref. [52] demonstrated that a spacecraft moves on a trajectory around the stable libration points for at least 700 days before the solar influence causes it to move through wide departures from the Lagrangian points. Indeed, from the analysis of the plots it does not appear that, after 700 days, a limiting value for the envelope is approached. It would be interesting to recover this feature directly from the solution of the dynamical equations (if they were known), since at the present state we believe that the form of the equations involved (see Sec. II) does not allow, by itself, such a deduction.

The first purpose of our paper consists in showing that this is true also if we assume the quantum corrected potential discovered in Refs. [5, 12], and Secs. II and III are devoted to this topic. All the considerations made in these Sections represent the natural extension of our previous papers, as we continue to describe the three-body problem in the context of effective field theories of gravity by adding all features that would contribute to make this subject as close as possible to reality, in order to encourage the launch of future space missions that could verify the model we are proposing. On the other hand, one has to consider 
that general relativity is currently the most successful gravitational theory describing the nature of space and time, and well confirmed by observations. In fact, it has been brightly confirmed by all the so-called "classical" tests, i.e. the perihelion shift of Mercury, the deflection of light and the Shapiro time delay, and it has also gone through the systematic test offered by the binary pulsar system "PSR $1913+16$ ", since the orbit decay of this system is perfectly in accordance with the theoretical decay due to the emission of gravitational waves, as predicted by general relativity. Furthermore, Lagrangian points have recently attracted renewed interests for relativistic astrophysics [53-56], where the position and the stability of Lagrangian points is described within the post-Newtonian regime. For all these reasons, we believe that our model is incomplete without a comparison with the Einstein theory. Therefore, Sec. IV studies all Lagrangian points within the framework of general relativity, to establish the most accurate classical counterpart of the putative quantum framework that we have set up. By taking seriously into account the important role played by the Einstein theory within this scheme, in the last part of this paper we describe a new quantum corrected regime where the underlying classical theory is represented by general relativity, rather than Newtonian theory. All the considerations made in Refs. [13-15], in fact, are characterized by the fact that the classical theory for which quantum corrections are computed is the Newtonian theory, instead of Einstein's one. But, if general relativity is the most successful classical theory of gravitation, then we have to consider a scheme where quantum corrections to general relativity are evaluated. This topic is investigated in Sec. V, where we also show that, among all quantum coefficients $\kappa_{1}$ and $\kappa_{2}$ in the long-distance corrections to the Newtonian potential available in literature [5, 12-15], the most suitable ones to describe the gravitational interactions involving (at least) three bodies are those connected to the bound-states potential. Finally, conclusions and open problems are discussed in Sec. VI.

\section{THE QUANTUM CORRECTED EQUATIONS OF THE RESTRICTED FOUR- BODY PROBLEM}

We start by introducing the classical dynamical equations governing the motion of the planetoid in the gravitational field of the Earth, the Moon and the Sun $[52,57]$. We suppose that the Earth and the Moon move in circular orbit around their mass center and the mass center, in turn, moves in circular orbit about the Sun. The Earth-Moon orbit plane is inclined 
at an angle $i=5^{\circ} 9^{\prime}$ to the plane of the ecliptic. We introduce the rotating coordinate system $\xi, \eta, \zeta$ with the Earth-Moon mass center as its origin and characterized by the fact that the $\xi$ axis lies along the Earth-Moon line, the $\eta$ axis lies in the Earth-Moon orbit plane and the $\zeta$ axis points in the direction of the angular velocity vector of the Earth-Moon configuration. The $\xi, \eta$ axes rotate about the $\zeta$ axis with the angular velocity $\omega$ of the Earth-Moon line. If the vector $\overrightarrow{\mathcal{R}}=(\xi, \eta, \zeta)$ indicates in this coordinate system the position of a spacecraft of infinitesimal mass, the vector dynamical equation describing its motion is

$$
\ddot{\overrightarrow{\mathcal{R}}}+\vec{\omega} \times(2 \dot{\overrightarrow{\mathcal{R}}}+\dot{\vec{\omega}} \times \overrightarrow{\mathcal{R}})=-\vec{\nabla}_{\mathcal{R}} V+\vec{\nabla}_{\mathcal{R}} U+\vec{S}
$$

where

$$
\begin{gathered}
V \equiv \frac{G m_{1}}{\rho_{1}}+\frac{G m_{2}}{\rho_{2}}, \\
U \equiv G m_{3}\left[\frac{1}{\rho_{3}}-\frac{\overrightarrow{\mathcal{R}} \cdot \overrightarrow{\mathcal{R}_{3}}}{\left(\mathcal{R}_{3}\right)^{3}}\right],
\end{gathered}
$$

with $G$ being the universal gravitation constant, $m_{1}, m_{2}$ and $m_{3}$ the mass of the Earth, the Moon and the Sun, respectively, $\rho_{1}, \rho_{2}$ and $\rho_{3}$ the distances from the planetoid of the Earth, the Moon and the Sun, respectively, $\mathcal{R}_{3}$ the distance of the Sun from the Earth-Moon mass center, and lastly $\vec{S}$ describes the solar radiation pressure. Written in components, Eq. (2.1) becomes

$$
\begin{aligned}
\ddot{\xi}-2 \omega \dot{\eta}-\omega^{2} \xi & =-\frac{\partial V}{\partial \xi}+\frac{\partial U}{\partial \xi}+S_{\xi}, \\
\ddot{\eta}+2 \omega \dot{\xi}-\omega^{2} \eta & =-\frac{\partial V}{\partial \eta}+\frac{\partial U}{\partial \eta}+S_{\eta}, \\
\ddot{\zeta} & =-\frac{\partial V}{\partial \zeta}+\frac{\partial U}{\partial \zeta}+S_{\zeta} .
\end{aligned}
$$

We can write Eqs (2.4)-(2.6) in what we denote by $x, y, z$ system, which is the rotating noninertial coordinate frame of reference centered at one of the two noncollinear Lagrangian points, e.g. $L_{4}$. If we use the transformations

$$
\begin{aligned}
\xi & =x+\xi_{p} \\
\eta & =y+\eta_{p} \\
\zeta & =z
\end{aligned}
$$


where $\xi_{p}$ and $\eta_{p}$ are the constant coordinates of the libration point $L_{4}$ in the $\xi, \eta, \zeta$ system, then Eqs. (2.4)-(2.6) become

$$
\begin{gathered}
\ddot{x}=2 \omega \dot{y}+\left(x+\xi_{p}\right) \omega^{2}-\left(x_{3}+\xi_{p}\right)\left(\Omega_{\omega}\right)^{2}+S_{x}+\sum_{i=1}^{3} \frac{G m_{i}}{\rho_{i}^{3}}\left(x_{i}-x\right), \\
\ddot{y}=-2 \omega \dot{x}+\left(y+\eta_{p}\right) \omega^{2}-\left(y_{3}+\eta_{p}\right)\left(\Omega_{\omega}\right)^{2}+S_{y}+\sum_{i=1}^{3} \frac{G m_{i}}{\rho_{i}^{3}}\left(y_{i}-y\right), \\
\ddot{z}=-z_{3}\left(\Omega_{\omega}\right)^{2}+S_{z}+\sum_{i=1}^{3} \frac{G m_{i}}{\rho_{i}^{3}}\left(z_{i}-z\right),
\end{gathered}
$$

where $\Omega_{\omega}$ is the angular velocity of the Earth-Moon mass center around the Sun, and the relation $G m_{3} /\left(\mathcal{R}_{3}\right)^{3}=\left(\Omega_{\omega}\right)^{2}$ has been exploited. Moreover, the distances $\rho_{i}$ are given by

$$
\left(\rho_{i}\right)^{2}=\left(x_{i}-x\right)^{2}+\left(y_{i}-y\right)^{2}+\left(z_{i}-z\right)^{2} \quad(i=1,2,3),
$$

where the coordinates $\left(x_{1}, y_{1}\right)$ and $\left(x_{2}, y_{2}\right)$ of the Earth and the Moon respectively are deduced from $(2.7)$ once the coordinates $\left(\xi_{p}, \eta_{p}\right)$ of $L_{4}$ are known (remember we have $z_{1}=$ $z_{2}=0$ ), whereas the coordinates of the Sun are given by the relations

$$
\begin{aligned}
& x_{3}=\mathcal{R}_{3}(\cos \psi \cos \theta+\cos i \sin \psi \sin \theta)-\xi_{p}, \\
& y_{3}=-\mathcal{R}_{3}(\cos \psi \sin \theta-\cos i \sin \psi \cos \theta)-\eta_{p}, \\
& z_{3}=\mathcal{R}_{3} \sin \psi \sin i
\end{aligned}
$$

where $\psi$ is the angular position of the Sun with respect to the vernal equinox and measured in the plane of the ecliptic, and $\theta$ describes the position of the Earth-Moon line with respect to the vernal equinox measured in the Earth-Moon orbit plane (see Fig. 2 of Ref. [52]). The relations defining these angles are

$$
\begin{aligned}
& \psi=\Omega_{\omega} t+\psi_{0}, \\
& \theta=\Omega_{\omega} t+\theta_{0},
\end{aligned}
$$

where $\psi_{0}$ and $\theta_{0}$ are the initial values of $\theta$ and $\psi$. For our computation we have used the following numerical values: $\Omega_{\omega}=1.99082 \times 10^{-7} \mathrm{rad} / \mathrm{s}, \omega=2.665075637 \times 10^{-6} \mathrm{rad} / \mathrm{s}$, $\psi_{0}=\theta_{0}=0$ (i.e. the initial position of the Sun will be on the extended Earth-Moon line, with the Moon in between Earth and Sun). Moreover, following Ref. [15], we have the classical values $\xi_{p}=1.87528148802 \times 10^{8} \mathrm{~m}$ and $\eta_{p}=3.32900165215 \times 10^{8} \mathrm{~m}$. If we initially 
set $\vec{S}=\overrightarrow{0}$ in (2.1), we obtain that the perturbative effect of the Sun makes the spacecraft ultimately escape from the stable equilibrium point after about 700 days [52], as is shown in Figs. 1 and 2. As can be noticed from Fig. 1, the irregular initial motion damps out

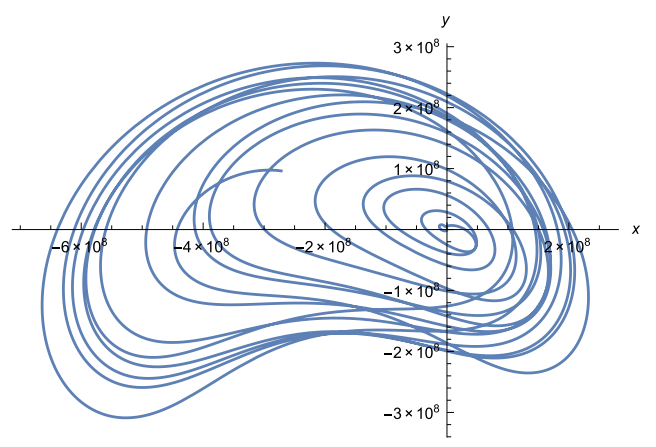

FIG. 1: Parametric plot of the spacecraft motion about $L_{4}$ resulting from zero initial displacement and velocity in the classical case. The quantities appearing on the axes are measured in meters and the time interval considered is about $4 \times 10^{7} \mathrm{~s}$.

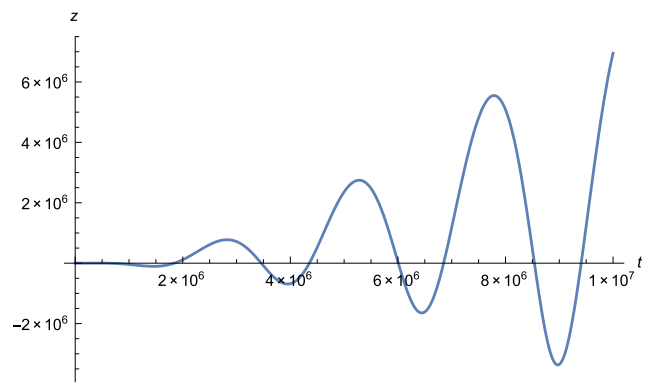

FIG. 2: Plot of the spacecraft motion about $L_{4}$ in the $z$-direction resulting from zero initial displacement and velocity in the classical case. The quantities on the axes are measured in meters and in seconds.

and there is an approximate one-month periodicity associated with the motion. Moreover, Fig. 2 shows that the amplitude of the motion increases with time and that the period of motion is about 27, 6 days, a value really near to the 29,53 days of the synodical month. All these results indicate that the spacecraft will escape from the equilibrium point $L_{4}$ (or equivalently $L_{5}$ ) or, in other words, the perturbing presence of the Sun makes the points $L_{4}$ and $L_{5}$ cease to be equilibrium points, but they are "stable" in the sense indicated in the Introduction.

All these considerations are valid within the classical scheme, whereas in the quantum 
corrected regime (see Secs. IV and V) the Newtonian potential is corrected by a Poincaré asymptotic expansion involving integer powers of $G$ only, so that Eq. (2.1) can be replaced by the vector dynamical equation

$$
\ddot{\overrightarrow{\mathcal{R}}}+\vec{\omega} \times(2 \dot{\overrightarrow{\mathcal{R}}}+\dot{\vec{\omega}} \times \overrightarrow{\mathcal{R}})=-\vec{\nabla}_{\mathcal{R}} V_{q}+\vec{\nabla}_{\mathcal{R}} U_{q}+\vec{S}
$$

with $[5,12-15]$

$$
\begin{gathered}
V_{q}=\frac{G m_{1}}{\rho_{1}}\left[1+\frac{k_{1}}{\rho_{1}}+\frac{k_{2}}{\left(\rho_{1}\right)^{2}}\right]+\frac{G m_{2}}{\rho_{2}}\left[1+\frac{k_{1}^{\prime}}{\rho_{2}}+\frac{k_{2}}{\left(\rho_{2}\right)^{2}}\right], \\
U_{q}=\frac{G m_{3}}{\rho_{3}}\left[1+\frac{k_{1}^{\prime \prime}}{\rho_{3}}+\frac{k_{2}}{\left(\rho_{3}\right)^{2}}\right]-G m_{3} \frac{\overrightarrow{\mathcal{R}} \cdot \overrightarrow{\mathcal{R}_{3}}}{\left(\mathcal{R}_{3}\right)^{3}}\left[1+\frac{2 k_{1}^{\prime \prime}}{\mathcal{R}_{3}}+\frac{3 k_{2}}{\left(\mathcal{R}_{3}\right)^{2}}\right],
\end{gathered}
$$

where, following Ref. [12], we decide to adopt the results concerning the bound-states potential. Even without knowing the detailed calculations of Sec. V, we may point out that, in classical gravity, the Levi-Civita cancellation theorem [58] holds, according to which the $N$-body Lagrangian in general relativity can be always reduced to a Lagrangian of $N$ point particles. In other words, it is not necessary to assume that we deal with point particles for simplicity, but the effects of their size get eventually and exactly cancelled. Now the quantum corrections considered in Refs. [13-15] deal precisely with the longdistance Newtonian potential among point particles, and consider three distinct physical settings: scattering, or bound states, or one-particle reducible [12]. We think that, in celestial mechanics, the bound states picture is more appropriate for studying stable and unstable equilibrium points. Therefore we set (cf. Sec. V)

$$
k_{1}=-\frac{G m_{1}}{2 c^{2}}, k_{1}^{\prime}=-\frac{G m_{2}}{2 c^{2}}, k_{1}^{\prime \prime}=-\frac{G m_{3}}{2 c^{2}}, k_{2}=\frac{41}{10 \pi}\left(l_{P}\right)^{2},
$$

$l_{P}$ being the Planck length. The occurrence of the term $k_{2}$, which is quadratic in the Planck length, cannot be obvious for the general reader, and hence we here summarize its properties and derivation, following our sources [5-7, 12]. The one-loop quantum correction to the gravitational potential is a low-energy property independent of the ultimate highenergy theory. The potential of gravitational scattering of two heavy masses turns out to be

$$
V(r)=-\frac{G M m}{r}\left[1+3 \frac{G(M+m)}{c^{2} r}+\frac{k_{2}}{r^{2}}\right] .
$$

From dimensional analysis one can indeed expect a term like $\frac{k_{2}}{r^{2}}$, because the unique dimensionless term linear in $\hbar$ and linear in $G$ is $\frac{G \hbar}{c^{3} r^{2}}$. The classical post-Newtonian correction is 
also a well-known dimensionless combination, without $\hbar$. We have a numerical factor of $-\frac{1}{2}$ in (2.17) obtained as $3-\frac{7}{2}=-\frac{1}{2}$, because the bound-state contribution [12] written within square brackets above is $-\frac{7}{2}$. By Fourier transform, the corresponding results in momentum space turn out to be [7]

$$
\frac{1}{r} \rightarrow \frac{1}{q^{2}}, \frac{1}{r^{2}} \rightarrow \frac{1}{q^{2}} \times \sqrt{q^{2}}, \frac{1}{r^{3}} \rightarrow \frac{1}{q^{2}} \times q^{2} \log \left(q^{2}\right), \delta^{3}(\vec{x}) \rightarrow \frac{1}{q^{2}} \times q^{2} .
$$

The one-loop potential is obtained from one-graviton exchange, with the $\frac{1}{q^{2}}$ resulting from the massless propagator. The corrections linear in $\hbar$ are due to all one-loop diagrams that can contribute to the scattering of two masses. The kinematic dependence of the loops then brings in nonanalytic corrections of the form $G m \sqrt{q^{2}}, G q^{2} \log \left(q^{2}\right)$, as well as analytic terms $G q^{2}$. However, the Fourier transform of the analytic term is a Dirac delta in position space, and hence analytic terms do not contribute to long-distance modifications of the potential. The above correspondences are made precise by the following integrals [12]:

$$
\int \frac{\mathrm{d}^{3} q}{(2 \pi)^{3}} \mathrm{e}^{\mathrm{i} \vec{q} \cdot \vec{r}} \frac{1}{|\vec{q}|^{2}}=\frac{1}{4 \pi r}, \int \frac{\mathrm{d}^{3} q}{(2 \pi)^{3}} \mathrm{e}^{\mathrm{i} \vec{q} \cdot \vec{r}} \frac{1}{|\vec{q}|}=\frac{1}{2 \pi^{2} r^{2}}, \int \frac{\mathrm{d}^{3} q}{(2 \pi)^{3}} \mathrm{e}^{\mathrm{i} \vec{q} \cdot \vec{r}} \log \left(|\vec{q}|^{2}\right)=-\frac{1}{2 \pi r^{3}} .
$$

In the $x, y, z$ system, instead of Eqs. (2.8)-(2.10), Eq. (2.14), written in components, gives rise to the system

$$
\begin{aligned}
\ddot{x} & =2 \omega \dot{y}+\left(x+\xi_{p}\right) \omega^{2}-\left(x_{3}+\xi_{p}\right)\left(\Omega_{\omega}\right)^{2}\left[1+\frac{2 k_{1}^{\prime \prime}}{\mathcal{R}_{3}}+\frac{3 k_{2}}{\left(\mathcal{R}_{3}\right)^{2}}\right]+\frac{G m_{1}\left(x_{1}-x\right)}{\left(\rho_{1}\right)^{3}}\left[1+\frac{2 k_{1}}{\rho_{1}}+\frac{3 k_{2}}{\left(\rho_{1}\right)^{2}}\right] \\
& +\frac{G m_{2}\left(x_{2}-x\right)}{\left(\rho_{2}\right)^{3}}\left[1+\frac{2 k_{1}^{\prime}}{\rho_{2}}+\frac{3 k_{2}}{\left(\rho_{2}\right)^{2}}\right]+\frac{G m_{3}\left(x_{3}-x\right)}{\left(\rho_{3}\right)^{3}}\left[1+\frac{2 k_{1}^{\prime \prime}}{\rho_{3}}+\frac{3 k_{2}}{\left(\rho_{3}\right)^{2}}\right]+S_{x}, \\
\ddot{y} & =-2 \omega \dot{x}+\left(y+\eta_{p}\right) \omega^{2}-\left(y_{3}+\eta_{p}\right)\left(\Omega_{\omega}\right)^{2}\left[1+\frac{2 k_{1}^{\prime \prime}}{\mathcal{R}_{3}}+\frac{3 k_{2}}{\left(\mathcal{R}_{3}\right)^{2}}\right]+\frac{G m_{1}\left(y_{1}-y\right)}{\left(\rho_{1}\right)^{3}}\left[1+\frac{2 k_{1}}{\rho_{1}}+\frac{3 k_{2}}{\left(\rho_{1}\right)^{2}}\right] \\
& +\frac{G m_{2}\left(y_{2}-y\right)}{\left(\rho_{2}\right)^{3}}\left[1+\frac{2 k_{1}^{\prime}}{\rho_{2}}+\frac{3 k_{2}}{\left(\rho_{2}\right)^{2}}\right]+\frac{G m_{3}\left(y_{3}-y\right)}{\left(\rho_{3}\right)^{3}}\left[1+\frac{2 k_{1}^{\prime \prime}}{\rho_{3}}+\frac{3 k_{2}}{\left(\rho_{3}\right)^{2}}\right]+S_{y}, \\
\ddot{z} & =-z_{3}\left(\Omega_{\omega}\right)^{2}\left[1+\frac{2 k_{1}^{\prime \prime}}{\mathcal{R}_{3}}+\frac{3 k_{2}}{\left(\mathcal{R}_{3}\right)^{2}}\right]-\frac{G m_{1} z}{\left(\rho_{1}\right)^{3}}\left[1+\frac{2 k_{1}}{\rho_{1}}+\frac{3 k_{2}}{\left(\rho_{1}\right)^{2}}\right]-\frac{G m_{2} z}{\left(\rho_{2}\right)^{3}}\left[1+\frac{2 k_{1}^{\prime}}{\rho_{2}}+\frac{3 k_{2}}{\left(\rho_{2}\right)^{2}}\right] \\
& +\frac{G m_{3}\left(z_{3}-z\right)}{\left(\rho_{3}\right)^{3}}\left[1+\frac{2 k_{1}^{\prime \prime}}{\rho_{3}}+\frac{3 k_{2}}{\left(\rho_{3}\right)^{2}}\right]+S_{z},
\end{aligned}
$$

where we have used the fact that $z_{1}=z_{2}=0$. Setting $\vec{S}=\overrightarrow{0}$, we have integrated Eqs. (2.18)(2.20) and we have discovered that the situation is almost the same as in the classical case (see Figs. 3 and 4), i.e. the planetoid is destined to run away from the triangular libration points in about 700 days. This means that, also within a quantum corrected scheme, the gravitational effect of the Sun spoils the equilibrium condition at $L_{4}$ and $L_{5}$. 


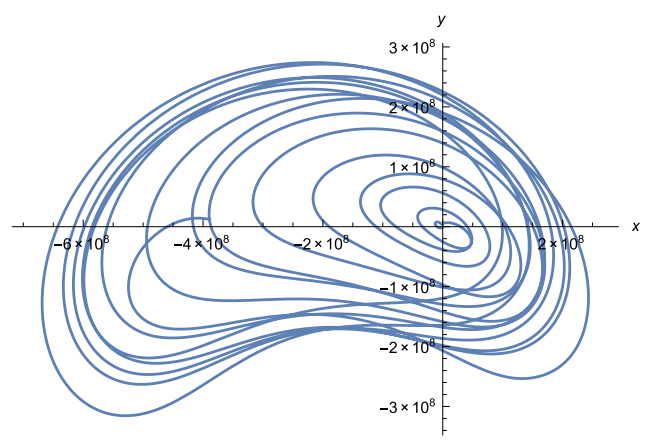

FIG. 3: Parametric plot of the spacecraft motion about $L_{4}$ resulting from zero initial displacement and velocity in the quantum case. The quantities appearing on the axes are measured in meters and the time interval considered is about $4 \times 10^{7} \mathrm{~s}$.

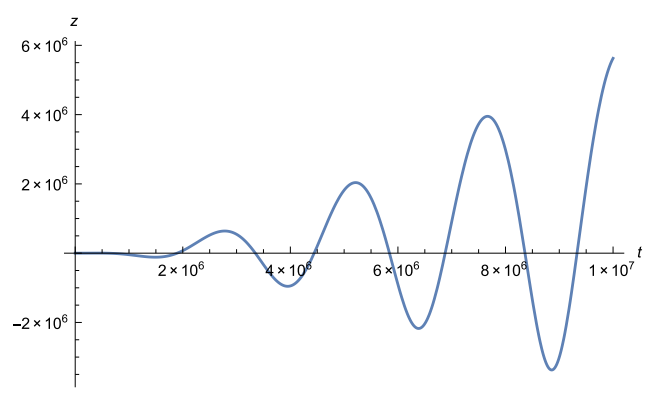

FIG. 4: Plot of the spacecraft motion about $L_{4}$ in the $z$-direction resulting from zero initial displacement and velocity in the quantum case. The quantities on the axes are measured in meters and in seconds.

\section{THE SOLAR RADIATION PRESSURE AND THE LINEAR STABILITY AT $L_{4}$}

At this stage, we assume the presence of the radiation pressure both in the classical equations (2.8)-(2.10) and in the quantum ones (2.18)-(2.20). The solar radiation pressure is given by

$$
\vec{S}=-K \frac{A}{m\left(\rho_{3}\right)^{3}} \vec{\rho}_{3}
$$

where $A$ is the cross-sectional area normal to $\vec{\rho}_{3}, m$ is the planetoid mass and $K$ is a constant. Inspired by Ref. [52], we use the value $K=2,048936 \times 10^{17} N$. We have integrated the classical equations (2.8)-(2.10) and we have found that the presence of the solar radiation pressure causes the vehicle to move further away from $L_{4}$ in a given time, as one can see from Fig. 5. In particular, the larger the ratio $A / m$ is, the larger the envelope of the motion 


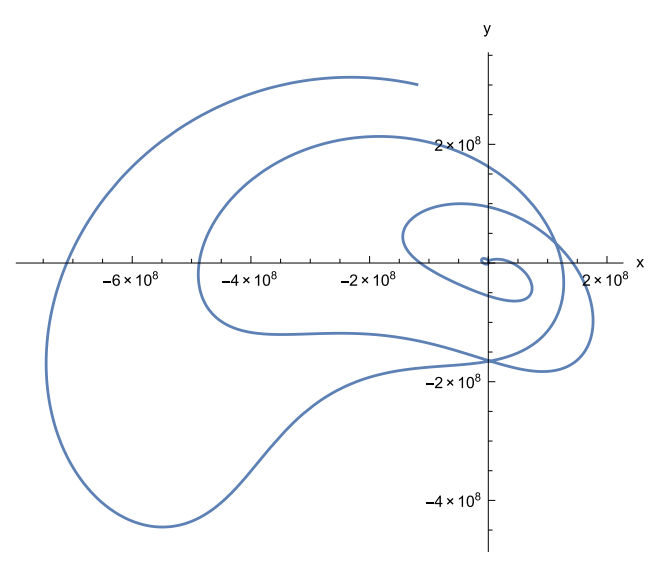

FIG. 5: Parametric plot in the classical regime of the spacecraft motion about $L_{4}$ in the presence of the solar radiation pressure and considering $A / m=0,159 \mathrm{~m}^{2} / \mathrm{Kg}$. The initial displacement and velocity are zero. The quantities appearing on the axes are measured in meters and the time interval considered is about $1 \times 10^{7} \mathrm{~s}$.

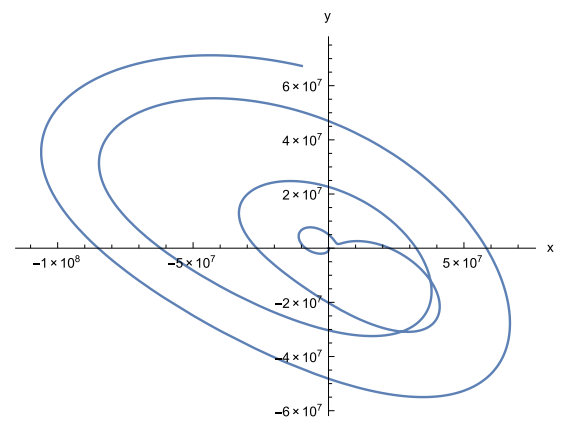

FIG. 6: Parametric plot in the quantum regime of the spacecraft motion about $L_{4}$ in the presence of the solar radiation pressure and considering $A / m=0,159 \mathrm{~m}^{2} / \mathrm{Kg}$. The initial displacement and velocity are zero. The quantities appearing on the axes are measured in meters and the time interval considered is about $1 \times 10^{7} \mathrm{~s}$.

is $[52]$.

Interestingly, in the quantum case ruled by effective gravity the situation is a little bit different. Unlike the classical regime, the presence of the solar radiation pressure in the equations (2.18)-(2.20) does not show itself through the fact that the spacecraft goes away from the triangular libration points more rapidly, but it results in a less chaotic and irregular motion about $L_{4}$, which ultimately make the planetoid escape from $L_{4}$, like in the classical 

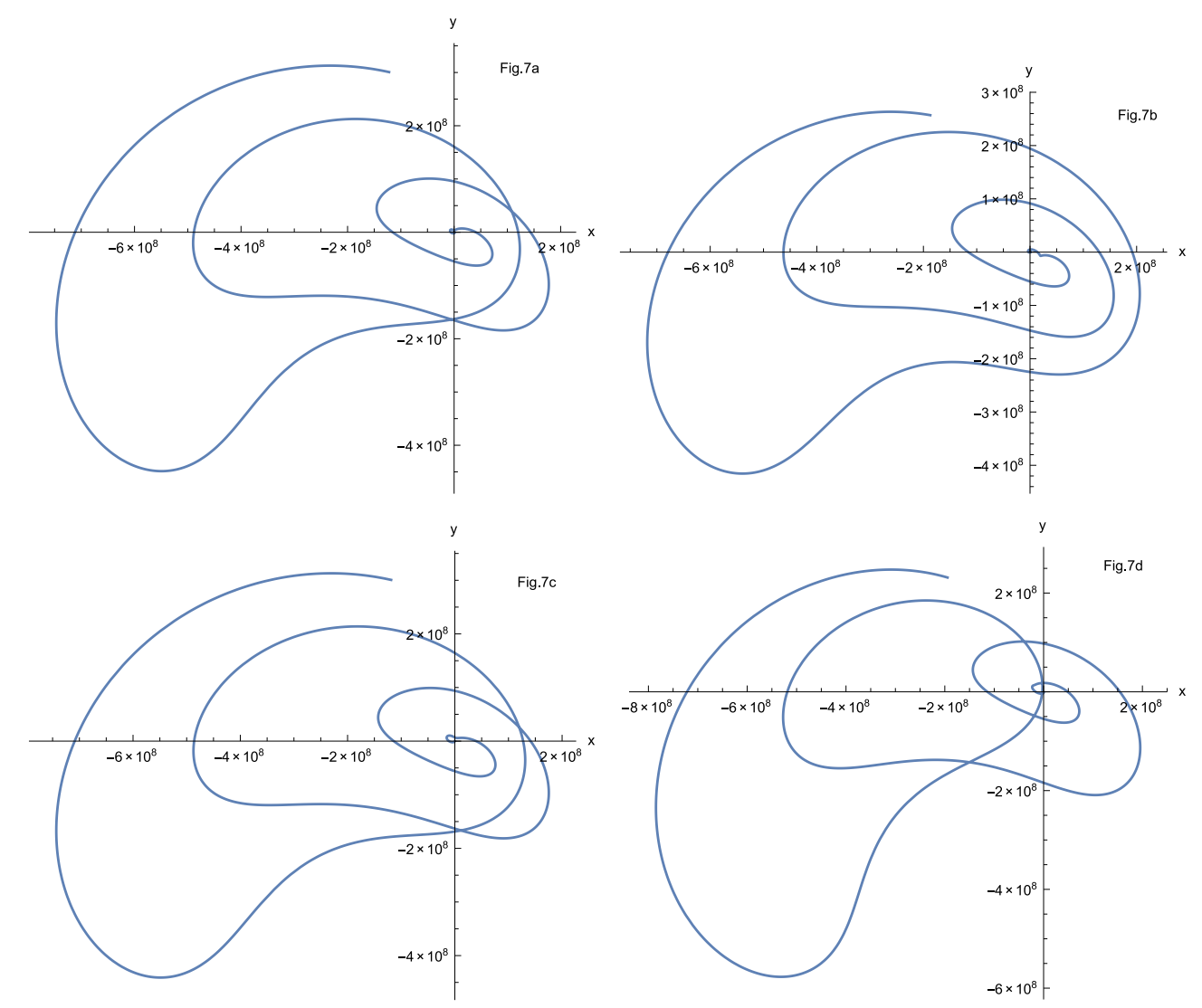

FIG. 7: Fig. 7a: Spacecraft motion about $L_{4}$ in the classical regime and with an initial velocity of $3 \mathrm{~m} / \mathrm{s}$ at $60^{\circ}$; Fig. 7b: Spacecraft motion about $L_{4}$ in the classical regime and with an initial velocity of $3 \mathrm{~m} / \mathrm{s}$ at $150^{\circ}$; Fig. $7 \mathrm{c}$ : Spacecraft motion about $L_{4}$ in the classical regime and with an initial velocity of $3 \mathrm{~m} / \mathrm{s}$ at $240^{\circ}$; Fig. $7 \mathrm{~d}$ : Spacecraft motion about $L_{4}$ in the classical regime and with an initial velocity of $3 \mathrm{~m} / \mathrm{s}$ at $330^{\circ}$.

case. These effects are clearly visible from Fig. $6^{1}$. We can also try to find the best set of initial conditions which leads to the smallest envelope of the motion of the planetoid. We have studied several sets of initial conditions both in the classical case and in the quantum one. In the classical regime, we completely agree with the results of Ref. [52]. We have found in fact that the amplitude of the spacecraft's motion depends strongly on the position of the Sun (i.e. on the values assumed by $\theta_{0}$ and $\psi_{0}$ ) and on its initial position and velocity. For example, Fig. 7 shows the motion resulting from an initial zero displacement and different

\footnotetext{
${ }^{1}$ The different scale adopted in Fig. 6 with respect to the one of Fig. 5 allows us to better appreciate its features.
} 
initial velocity $\left(\theta_{0}=\psi_{0}=0\right)$ and the time interval considered is about $1 \times 10^{7} \mathrm{~s}$. As we can see, the envelope of the motion in Fig. $7 \mathrm{~b}$ is smaller at any time than the envelope of the motion shown in Fig. 5 .

The situation is fairly the same in the quantum regime (Fig. 8), where we have discovered that one set of initial conditions (having $\theta_{0}=\psi_{0}=0$ ) exists which results in a smaller envelope of the spacecraft motion at any given time, as one can see from Fig. 8b. This fact can be understood with a comparison between Figs. 6 and 8b. The interesting difference with respect to the classical case consists in the fact that the reduction of the envelope of the planetoid motion produced by a nonzero initial velocity becomes more evident in the quantum regime. By inspection of Figs. 7 and 8 we discover a strong dependence on the initial conditions of the planetoid trajectories both in the classical and quantum regime. This suggests that, from an experimental point of view, it might be useful to drop off two or more satellites close to the Lagrangian points $L_{4}$ and $L_{5}$ with slightly different initial conditions for position and velocity. Measurements of the satellite differential positions, together with the measurement of the single orbits, could make it possible to discriminate between classical and quantum regime, without depending on the absolute knowledge of Lagrangian points' location.

If we want to force the particle to stay precisely at $L_{4}$, we have to set aside the perturbing force due to the Sun by the application of a continuous force (see Fig. 9). Therefore, we have to study the following stability equation (in the $\xi, \eta, \zeta$ system):

$$
-\vec{\nabla}_{\mathcal{R}} V+\vec{\nabla}_{\mathcal{R}} U+\vec{S}+\frac{\vec{F}}{m}=\overrightarrow{0},
$$

which becomes in the quantum case

$$
-\vec{\nabla}_{\mathcal{R}} V_{q}+\vec{\nabla}_{\mathcal{R}} U_{q}+\vec{S}+\frac{\vec{F}_{q}}{m}=\overrightarrow{0}
$$

where $m$ is the mass of the planetoid and $\vec{F}$ (respectively, $\vec{F}_{q}$ ) represents the force to be applied to the spacecraft in order to make it stay precisely at $L_{4}$ in the classical (respectively, quantum) regime. If we consider Eqs. (3.2)-(3.3) in the $x, y, z$ coordinate system, we can exploit the simplification resulting from the fact that the planetoid must be at the position $x=y=z=0$, hence Eq. (3.2), written in components, becomes

$$
\xi_{p} \omega^{2}-\left(x_{3}+\xi_{p}\right)\left(\Omega_{\omega}\right)^{2}+S_{x}+\sum_{i=1}^{3} \frac{G m_{i}}{\rho_{i}^{3}} x_{i}+\frac{F_{x}}{m}=0,
$$



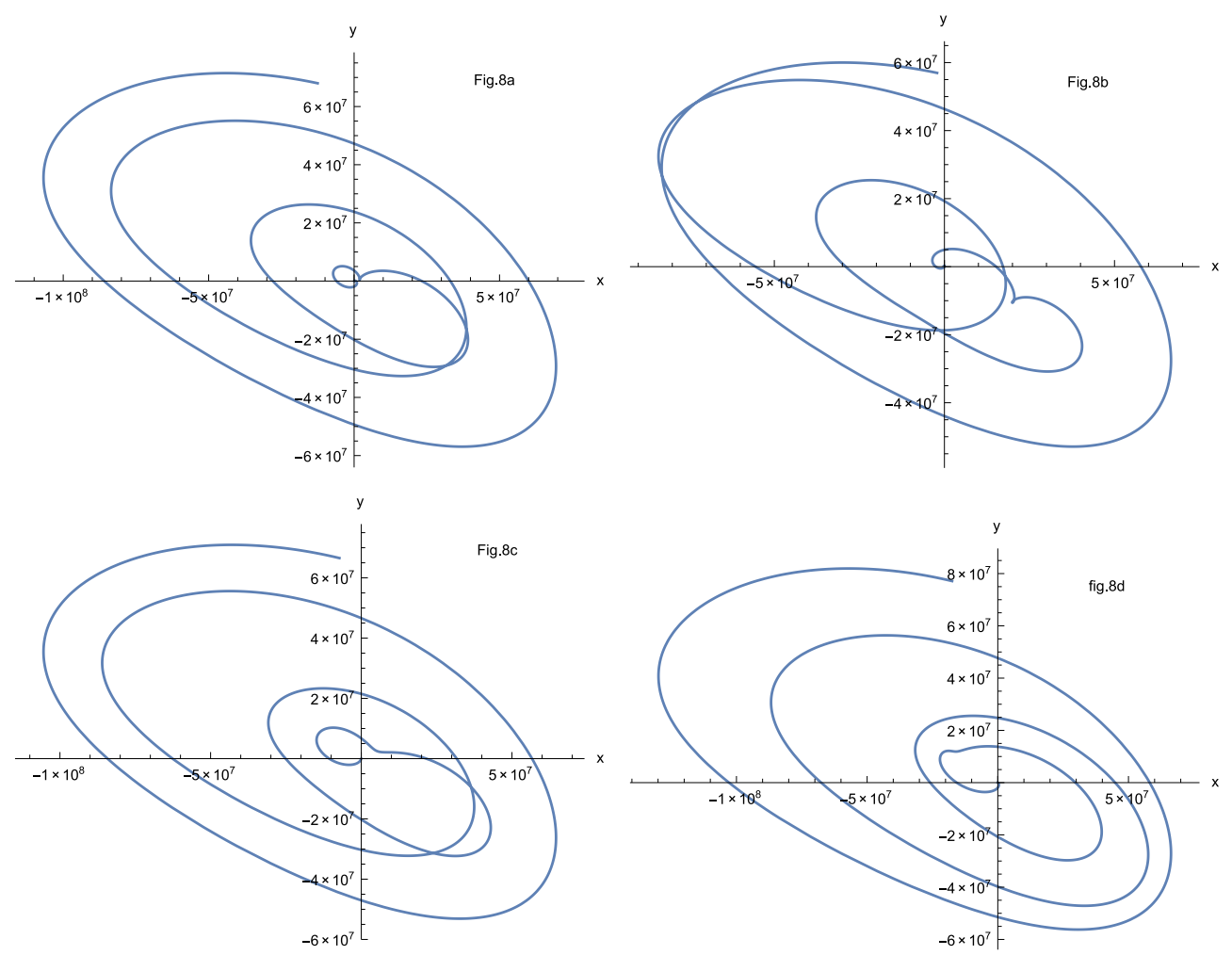

FIG. 8: Fig. 8a: Spacecraft motion about $L_{4}$ in the quantum regime and with an initial velocity of $3 \mathrm{~m} / \mathrm{s}$ at $60^{\circ}$; Fig. $8 \mathrm{~b}$ : Spacecraft motion about $L_{4}$ in the quantum regime and with an initial velocity of $3 \mathrm{~m} / \mathrm{s}$ at $150^{\circ}$; Fig. 8 c: Spacecraft motion about $L_{4}$ in the quantum regime and with an initial velocity of $3 \mathrm{~m} / \mathrm{s}$ at $240^{\circ}$; Fig. 8 d: Spacecraft motion about $L_{4}$ in the quantum regime and with an initial velocity of $3 \mathrm{~m} / \mathrm{s}$ at $330^{\circ}$.
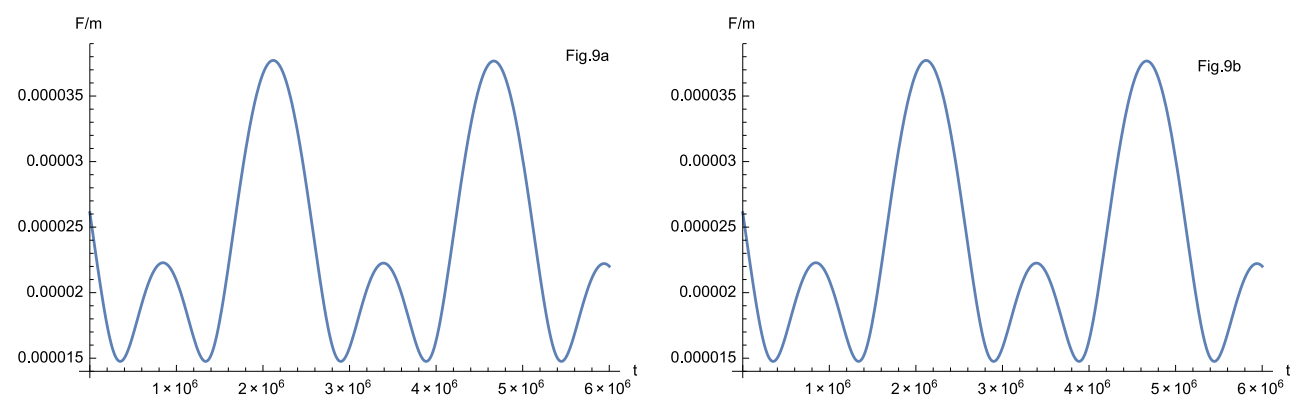

FIG. 9: Fig. 9a: Force per unit mass as a function of time required to induce stability at $L_{4}$ in the classical regime; Fig. 9b: Force per unit mass as a function of time required to induce stability at $L_{4}$ in the quantum regime. 


$$
\begin{gathered}
\eta_{p} \omega^{2}-\left(y_{3}+\eta_{p}\right)\left(\Omega_{\omega}\right)^{2}+S_{y}+\sum_{i=1}^{3} \frac{G m_{i}}{\rho_{i}^{3}} y_{i}+\frac{F_{y}}{m}=0 \\
-z_{3}\left(\Omega_{\omega}\right)^{2}+S_{z}+\frac{G m_{3}}{\rho_{i}^{3}} z_{3}+\frac{F_{z}}{m}=0
\end{gathered}
$$

whereas from Eq. (3.3) we obtain

$$
\begin{gathered}
\xi_{p} \omega^{2}-\left(x_{3}+\xi_{p}\right)\left(\Omega_{\omega}\right)^{2}\left[1+\frac{2 k_{1}^{\prime \prime}}{\mathcal{R}_{3}}+\frac{3 k_{2}}{\left(\mathcal{R}_{3}\right)^{2}}\right]+\frac{G m_{1} x_{1}}{\left(\rho_{1}\right)^{3}}\left[1+\frac{2 k_{1}}{\rho_{1}}+\frac{3 k_{2}}{\left(\rho_{1}\right)^{2}}\right] \\
+\frac{G m_{2} x_{2}}{\left(\rho_{2}\right)^{3}}\left[1+\frac{2 k_{1}^{\prime}}{\rho_{2}}+\frac{3 k_{2}}{\left(\rho_{2}\right)^{2}}\right]+\frac{G m_{3} x_{3}}{\left(\rho_{3}\right)^{3}}\left[1+\frac{2 k_{1}^{\prime \prime}}{\rho_{3}}+\frac{3 k_{2}}{\left(\rho_{3}\right)^{2}}\right]+S_{x}+\frac{F_{q_{x}}}{m}=0, \\
\eta_{p} \omega^{2}-\left(y_{3}+\eta_{p}\right)\left(\Omega_{\omega}\right)^{2}\left[1+\frac{2 k_{1}^{\prime \prime}}{\mathcal{R}_{3}}+\frac{3 k_{2}}{\left(\mathcal{R}_{3}\right)^{2}}\right]+\frac{G m_{1} y_{1}}{\left(\rho_{1}\right)^{3}}\left[1+\frac{2 k_{1}}{\rho_{1}}+\frac{3 k_{2}}{\left(\rho_{1}\right)^{2}}\right] \\
+\frac{G m_{2} y_{2}}{\left(\rho_{2}\right)^{3}}\left[1+\frac{2 k_{1}^{\prime}}{\rho_{2}}+\frac{3 k_{2}}{\left(\rho_{2}\right)^{2}}\right]+\frac{G m_{3} y_{3}}{\left(\rho_{3}\right)^{3}}\left[1+\frac{2 k_{1}^{\prime \prime}}{\rho_{3}}+\frac{3 k_{2}}{\left(\rho_{3}\right)^{2}}\right]+S_{y}+\frac{F_{q_{y}}}{m}=0, \\
-z_{3}\left(\Omega_{\omega}\right)^{2}\left[1+\frac{2 k_{1}^{\prime \prime}}{\mathcal{R}_{3}}+\frac{3 k_{2}}{\left(\mathcal{R}_{3}\right)^{2}}\right]+\frac{G m_{3} z_{3}}{\left(\rho_{3}\right)^{3}}\left[1+\frac{2 k_{1}^{\prime \prime}}{\rho_{3}}+\frac{3 k_{2}}{\left(\rho_{3}\right)^{2}}\right]+S_{z}+\frac{F_{q_{z}}}{m}=0 .
\end{gathered}
$$

Equations (3.4)-(3.6) and (3.7)-(3.9) make it possible for us to evaluate both the classical and the quantum force needed for stability and therefore the impulse per unit mass which the planetoid must be subjected to in order to stay in equilibrium exactly at $L_{4}$. Bearing in mind that the impulse is defined as the integral of a force over the time interval for which it acts, and on considering a time interval of one year, we have found the following results for the classical and the quantum regime, respectively:

$$
\begin{aligned}
& I_{c} / m=747,608255 \mathrm{~N} \mathrm{~s} / \mathrm{Kg}, \\
& I_{q} / m=747,608245 \mathrm{~N} \mathrm{~s} / \mathrm{Kg} .
\end{aligned}
$$

Of course, these considerations are preliminary because, even just at classical level, the fourbody problem has only been studied from us within Newtonian gravity. We also note that this calculation suggests a gedanken experiment in which two satellites are sent to $L_{4}$ and $L_{5}$, respectively. If the first satellite receives the impulse $I_{c}$ while the second receives the impulse $I_{q}$, one might try to check, by direct comparison, which value is better suited for stabilizing the Lagrangian point, gaining support for classical or, instead, quantum theory. However, this configuration is merely ideal because, in light of the very small relative difference of the impulse in the two cases, it looks practically impossible to keep all the experimental conditions (satellite mass, actuator and readout calibration, initial conditions, solar radiation pressure etc.) identical within the required accuracy (less than 0.1 parts per million). 


\section{THEORETICAL PREDICTIONS OF GENERAL RELATIVITY}

\section{A. Noncollinear Lagrangian points}

The analysis of the previous section relies on the simple but nontrivial assumption that, since effective gravity modifies the long-distance Newtonian potential among bodies of masses $m_{A}$ and $m_{B}$ according to ${ }^{2}$

$$
\begin{aligned}
V_{E}(r) & \sim-\frac{G m_{A} m_{B}}{r}\left[1+\left(\kappa_{1} \frac{\left(R_{A}+R_{B}\right)}{r}+\kappa_{2} \frac{\left(l_{P}\right)^{2}}{r^{2}}+\mathrm{O}\left(G^{2}\right)\right)\right] \\
\Longrightarrow & \frac{V_{E}(r)}{c^{2} m_{B}} \sim-\frac{R_{A}}{r}\left[1+\left(\kappa_{1} \frac{\left(R_{A}+R_{B}\right)}{r}+\kappa_{2} \frac{\left(l_{P}\right)^{2}}{r^{2}}+\mathrm{O}\left(G^{2}\right)\right)\right],
\end{aligned}
$$

for all values of $r$ greater than a suitably large $r_{0}$, where gravitational radii $R_{A}, R_{B}$ and Planck length $l_{P}$ are defined by

$$
R_{A} \equiv \frac{G m_{A}}{c^{2}}, R_{B} \equiv \frac{G m_{B}}{c^{2}}, l_{P} \equiv \sqrt{\frac{G \hbar}{c^{3}}},
$$

the resulting modification of Newtonian dynamics can be obtained by considering a classical Lagrangian where the Newtonian potential

$$
V_{N}(r)=-\frac{G m_{A} m_{B}}{r}
$$

is replaced by $V_{E}(r)$, while all other terms remain unaffected (cf. Sec. V). Although it would be inappropriate to use the quantum effective action to study the low-energy effects resulting from the asymptotic expansion (4.1), the above assumption is a shortcut to describe a theory lying in between classical gravity and full quantum gravity. For this reason, it becomes important to study the predictions of classical gravity when general relativity is instead assumed. The work in Ref. [55] has indeed done so by relying upon the Einstein-InfeldHoffmann equations of motion for a three-body system, but without studying an effective potential and the zeros of its gradient. However, such a potential is by now available in the literature, and the resulting approximate evaluation of Lagrangian points $L_{4}$ and $L_{5}$ was performed in Ref. [59], while their stability in a suitable mass range was proved in Ref. [60]. Strictly speaking, in general relativity the libration points become quasi-libration points,

\footnotetext{
${ }^{2}$ We can say that the real number $\kappa_{1}$ is the effective-gravity weight of the sum of gravitational radii, whereas the real number $\kappa_{2}$ is the effective-gravity weight of Planck's length squared.
} 
and we refer the reader to Ref. [61] for this feature, which reflects the expected emission of gravitational radiation.

For our purposes, it is enough to consider the relativistic version of the circular restricted three-body problem in a plane, where, for primaries of masses $\alpha$ and $\beta$ separated by a distance $l$, with gravitational radii $R_{\alpha} \equiv \frac{G \alpha}{c^{2}}$ and $R_{\beta} \equiv \frac{G \beta}{c^{2}}$, and mass ratio $\rho \equiv \frac{\beta}{\alpha}<1$, the classical angular frequency (or pulsation) $\omega \equiv \sqrt{\frac{G(\alpha+\beta)}{l^{3}}}$ is replaced by [59]

$$
\Omega \equiv \omega\left[1-\frac{3}{2} \frac{\left(R_{\alpha}+R_{\beta}\right)}{l}\left(1-\frac{1}{3} \frac{\rho}{(1+\rho)^{2}}\right)\right],
$$

while, in a noninertial frame with origin at the mass center of the Earth-Moon system, the equations of motion of the planetoid in the planar case (with coordinates $(\xi, \eta)$ ) read as $[59]$

$$
\begin{aligned}
& \ddot{\xi}-2 \Omega \dot{\eta}=\frac{\partial W}{\partial \xi}-\frac{\mathrm{d}}{\mathrm{d} t}\left(\frac{\partial W}{\partial \dot{\xi}}\right), \\
& \ddot{\eta}+2 \Omega \dot{\xi}=\frac{\partial W}{\partial \eta}-\frac{\mathrm{d}}{\mathrm{d} t}\left(\frac{\partial W}{\partial \dot{\eta}}\right),
\end{aligned}
$$

where, upon denoting by $r$ the distance of the planetoid from the primary of mass $\alpha$ (i.e. the Earth), and by $s$ the distance of the planetoid from the primary of mass $\beta$ (i.e. the Moon), given by

$$
\begin{aligned}
& r^{2}=\left(\xi+\frac{\rho l}{(1+\rho)}\right)^{2}+\eta^{2}, \\
& s^{2}=\left(\xi-\frac{l}{(1+\rho)}\right)^{2}+\eta^{2},
\end{aligned}
$$

one has the effective potential reading as [59]

$$
\begin{aligned}
W & =\frac{\Omega^{2}}{2}\left(\xi^{2}+\eta^{2}\right)+c^{2}\left[\frac{R_{\alpha}}{r}+\frac{R_{\beta}}{s}-\frac{1}{2}\left(\frac{\left(R_{\alpha}\right)^{2}}{r^{2}}+\frac{\left(R_{\beta}\right)^{2}}{s^{2}}\right)\right] \\
& +\frac{1}{8 c^{2}} f^{2}(\xi, \eta, \dot{\xi}, \dot{\eta})+\frac{3}{2}\left(\frac{R_{\alpha}}{r}+\frac{R_{\beta}}{s}\right) f(\xi, \eta, \dot{\xi}, \dot{\eta}) \\
& +\frac{R_{\beta}}{(1+\rho)} \Omega l\left(4 \dot{\eta}+\frac{7}{2} \Omega \xi\right)\left(\frac{1}{r}-\frac{1}{s}\right) \\
& +\frac{R_{\beta}}{(1+\rho)} \Omega^{2} l^{2}\left[-\frac{\eta^{2}}{2(1+\rho)}\left(\frac{\rho}{r^{3}}+\frac{1}{s^{3}}\right)-\frac{l}{r s}+\frac{(\rho-2)}{2(1+\rho)} \frac{1}{r}+\frac{(1-2 \rho)}{2(1+\rho)} \frac{1}{s}\right],
\end{aligned}
$$

where [59]

$$
f(\xi, \eta, \dot{\xi}, \dot{\eta}) \equiv \dot{\xi}^{2}+\dot{\eta}^{2}+2 \Omega(\xi \dot{\eta}-\eta \dot{\xi})+\Omega^{2}\left(\xi^{2}+\eta^{2}\right)
$$

At all equilibrium points, the first and second time derivatives of coordinates $(\xi, \eta)$ should vanish, which implies that it is enough to evaluate the zeros of the gradient of $W(\xi, \eta)$, 
because [59]

$$
\frac{\mathrm{d}}{\mathrm{d} t}\left(\frac{\partial W}{\partial \dot{\xi}}\right)=\frac{\mathrm{d}}{\mathrm{d} t}\left(\frac{\partial W}{\partial \dot{\eta}}\right)=0 \text { if } \dot{\xi}=\dot{\eta}=\ddot{\xi}=\ddot{\eta}=0 .
$$

Note now that, by virtue of (4.7) and (4.8), one has the formulas (A1)-(A4) in the Appendix, and hence the two components of the gradient can be expressed in the form

$$
\begin{gathered}
\frac{\partial W}{\partial \xi}=W_{1}(\xi, \eta, r)+W_{2}(\xi, \eta, s)+\frac{R_{\beta} l^{3}}{(1+\rho)} \frac{\Omega^{2}}{r s}\left[\xi\left(\frac{1}{r^{2}}+\frac{1}{s^{2}}\right)+\frac{l}{(1+\rho)}\left(\frac{\rho}{r^{2}}-\frac{1}{s^{2}}\right)\right], \\
\frac{\partial W}{\partial \eta}=\eta\left[W_{3}(\xi, \eta, r)+W_{4}(\xi, \eta, s)+\frac{R_{\beta} l^{3}}{(1+\rho)} \frac{\Omega^{2}}{r s}\left(\frac{1}{r^{2}}+\frac{1}{s^{2}}\right)\right],
\end{gathered}
$$

where the functions $W_{1}, \ldots, W_{4}$ are defined in Eqs. (A5)-(A8). Thus, unlike the case of Refs. [13-15], when the gradient of $w$ is set to zero with $\eta \neq 0$, one does not get an algebraic equation for $r$ only. Since we are interested in numerical solutions of such an enlarged algebraic system with (at least) ten decimal digits, we set $r \equiv \gamma l, s=\Gamma l$, and we study the coupled algebraic equations for the real numbers $\gamma$ and $\Gamma$ obtained from

$$
\begin{gathered}
\gamma^{5} \Gamma^{5} \frac{\partial W}{\partial \xi}=0, \\
\gamma^{5} \Gamma^{5} \frac{1}{\eta} \frac{\partial W}{\partial \eta}=0,
\end{gathered}
$$

where the fifth powers of $\gamma$ and $\Gamma$ are suggested by the occurrence of terms proportional to $\gamma^{-5}$ and $\Gamma^{-5}$ in the derivatives $\frac{\partial W}{\partial \xi}$ and $\frac{\partial W}{\partial \eta}$. We can write Eqs. (4.14)-(4.15) in a more concise way, i.e.

$$
\begin{gathered}
\gamma^{5} \Gamma^{5} \frac{\partial W}{\partial \xi}=\sum_{n=0}^{5} A_{n}\left(\Gamma^{j}\right) \gamma^{n}=0, \quad j \in\{0,1,2,3,4,5\}, \\
\gamma^{5} \Gamma^{5} \frac{1}{\eta} \frac{\partial W}{\partial \eta}=\sum_{n=0}^{5} B_{n}\left(\Gamma^{j}\right) \gamma^{n}=0, \quad j \in\{0,1,2,3,4,5\},
\end{gathered}
$$

where the coefficients $A_{n}\left(\Gamma^{j}\right)$ are given by

$$
\begin{aligned}
& A_{5}\left(\Gamma^{j}\right) \equiv \Gamma^{5}\left[1+\frac{\Omega^{2}}{2 c^{2}}\left(\eta^{2}+\xi^{2}\right)\right] \xi \Omega^{2}+\Gamma^{4}\left[\frac{3 \xi}{l}-\frac{7}{2(1+\rho)}\right] R_{\beta} \Omega^{2} \\
&+ \Gamma^{2}\left\{\frac{1}{2} \Omega^{2}\left[\frac{7 l \xi}{(1+\rho)}+\frac{l^{2}(2 \rho-1)}{(1+\rho)^{2}}-3\left(\eta^{2}+\xi^{2}\right)\right]-c^{2}\right\}\left(\xi-\frac{l}{(1+\rho)}\right) \frac{R_{\beta}}{l^{3}} \\
&+ {\left[\Gamma\left(\frac{c R_{\beta}}{l^{2}}\right)^{2}+\frac{3}{2} \frac{R_{\beta} \eta^{2}}{(1+\rho)^{2}} \frac{\Omega^{2}}{l^{3}}\right]\left(\xi-\frac{l}{(1+\rho)}\right), } \\
& A_{4}\left(\Gamma^{j}\right) \equiv \Gamma^{5}\left(\frac{3 \xi R_{\alpha} \Omega^{2}}{l}\right)+\Gamma^{2}[\xi(1+\rho)-l] \frac{\Omega^{2} R_{\beta}}{l(1+\rho)^{2}}
\end{aligned}
$$




$$
A_{3}\left(\Gamma^{j}\right)=0
$$

$$
\begin{gathered}
A_{2}\left(\Gamma^{j}\right) \equiv-\Gamma^{5}\left\{2 c^{2} R_{\alpha}(1+\rho)^{2}+\Omega^{2}\left[7 l R_{\beta} \xi(1+\rho)+3 R_{\alpha}\left(\eta^{2}+\xi^{2}\right)(1+\rho)^{2}+l^{2} R_{\beta}(\rho-2)\right]\right\} \\
\times \frac{[\xi+\rho(l+\xi)]}{2 l^{3}(1+\rho)^{3}}+\Gamma^{4}\left[2 l^{2} R_{\beta}(1+\rho)\right] \frac{\Omega^{2}[\xi+\rho(l+\xi)]}{2 l^{3}(1+\rho)^{3}} \\
A_{1}\left(\Gamma^{j}\right) \equiv \Gamma^{5}\left(\xi+l \frac{\rho}{(1+\rho)}\right)\left(\frac{c R_{\alpha}}{l^{2}}\right)^{2} \\
A_{0}\left(\Gamma^{j}\right) \equiv \Gamma^{5}[\xi+(l+\xi) \rho] \frac{3 R_{\alpha} \rho \eta^{2} \Omega^{2}}{2 l^{3}(1+\rho)^{3}}
\end{gathered}
$$

whereas the coefficients $B_{n}\left(\Gamma^{j}\right)$ are defined by

$$
\begin{aligned}
& B_{5}\left(\Gamma^{j}\right) \equiv \Gamma^{5}\left[2 c^{2}+\left(\eta^{2}+\xi^{2}\right) \Omega^{2}\right] \frac{\Omega^{2}}{2 c^{2}}+\Gamma^{4}\left(\frac{3 R_{\beta} \Omega^{2}}{l}\right) \\
&+\Gamma^{2}\left\{-(1+\rho)^{2}\left[2 c^{2}+3 \Omega^{2}\left(\eta^{2}+\xi^{2}\right)\right]+\Omega^{2}\left[7 l \xi(1+\rho)+l^{2}(2 \rho-3)\right]\right\} \\
& \times \frac{R_{\beta}}{2 l^{3}(1+\rho)^{2}}+\Gamma\left(\frac{c R_{\beta}}{l^{2}}\right)^{2}+\frac{3 R_{\beta} \eta^{2} \Omega^{2}}{2 l^{3}(1+\rho)^{2}}, \\
& B_{4}\left(\Gamma^{j}\right) \equiv \Gamma^{5}\left(\frac{3 R_{\alpha} \Omega^{2}}{l}\right)+\Gamma^{2}\left[\frac{R_{\beta} \Omega^{2}}{l(1+\rho)}\right] B_{3}\left(\Gamma^{j}\right)=0, \\
& B_{2}\left(\Gamma^{j}\right) \equiv-\Gamma^{5}\left\{\frac{c^{2} R_{\alpha}}{l^{3}}+\Omega^{2}\left[\frac{7 R_{\beta} \xi}{2 l^{2}(1+\rho)}+\frac{3 R_{\alpha}\left(\eta^{2}+\xi^{2}\right)}{2 l^{3}}-\frac{R_{\beta}}{l(1+\rho)^{2}}+\frac{3 R_{\beta} \rho}{2 l(1+\rho)^{2}}\right]\right\} \\
&+\Gamma^{4}\left[\frac{\Omega^{2} R_{\beta}}{l(1+\rho)}\right],
\end{aligned}
$$

The planetoid coordinates are eventually expressed, from the definitions (4.7) and (4.8), in the form

$$
\begin{gathered}
\xi=\frac{l}{2}\left[\left(\gamma^{2}-\Gamma^{2}\right)+\frac{(1-\rho)}{(1+\rho)}\right] \\
\eta= \pm l \sqrt{\gamma^{2}-\frac{1}{4}\left(\gamma^{2}-\Gamma^{2}+1\right)^{2}} .
\end{gathered}
$$

By numerical analysis of Eqs. (4.16)-(4.17) we have found that, in the Earth-Moon system, the only solution where both $\gamma$ and $\Gamma$ are different from zero is given by

$$
\gamma=0.99999999999996386756, \Gamma=0.99999999999284192083 .
$$


These values lead to a tiny departure from the equilateral triangle picture of Newtonian theory (this effect was first predicted in Ref. [62]), but less pronounced than in our earlier work [15], where we found a correction of $8.7894 \mathrm{~mm}$ on the $\xi$-coordinate and of $-4 \mathrm{~mm}$ on the $\eta$-coordinate [15]. We now find instead, for the planar coordinates of $L_{4}$,

$$
\xi^{(G R)}-\xi^{(N)}=2.73 \mathrm{~mm}, \eta^{(G R)}-\eta^{(N)}=-1.59 \mathrm{~mm} .
$$

At this stage, we can compare these corrections with those obtained through the method outlined by the authors of Ref. [55], where the position of the Lagrangian points is obtained by employing the Einstein-Infeld-Hoffman equation of motion rather than the analysis of the zeros of the gradient of the effective potential $w$ in (4.9). As we can see, the correction on the $\xi$-coordinate has got the same sign and the same magnitude as the one obtained with the method of Ref. [55], while the correction on the $\eta$-coordinate has got only the same sign, because the magnitude is three times bigger. Therefore, it is interesting to note the fact that two different methods give exactly the same correction of the $\xi$-coordinate. By taking account of Eq. (4.33), the resulting values of planetoid distance from Earth and Moon turn out to be

$$
r=\gamma l=3.8439999999998611069 \times 10^{8} \mathrm{~m}, s=\Gamma l=3.8439999999724843437 \times 10^{8} \mathrm{~m} .
$$

\section{B. Collinear Lagrangian points}

The position of the collinear Lagrangian points $L_{1}, L_{2}$ and $L_{3}$ is described by the system of equations

$$
\left\{\begin{array}{c}
\frac{\partial W}{\partial \xi}=0 \\
\eta=0
\end{array}\right.
$$

Following Ref. [15], we know that the vanishing of the $\eta$-coordinate implies that

$$
\xi=\epsilon r-l \frac{\rho}{(1+\rho)}, \quad(\epsilon= \pm 1),
$$

which in turn leads to the condition

$$
s= \pm(r-\epsilon l) .
$$

If we substitute relations (4.36)-(4.37) into Eq. (4.12) and initially adopt the choice $s=$ $(r-\epsilon l)$, we obtain an algebraic tenth degree equation where the only unknown is the distance 
$r$ of the planetoid from the Earth. By setting, as before, $r=\gamma l$, this equation can be written down as

$$
\sum_{n=0}^{10} C_{n} \gamma^{n}=0
$$

where

$$
\begin{aligned}
& C_{10} \equiv 1 \\
& C_{9} \equiv-\frac{(7 \rho+4)}{\epsilon(1+\rho)} \\
& C_{8} \equiv \frac{2 c^{2}}{\Omega^{2} l^{2}}+\frac{3\left(7 \rho^{2}+8 \rho+2\right)}{(1+\rho)^{2}}, \\
& C_{7} \equiv-\frac{1}{\epsilon(1+\rho)}\left\{\frac{c^{2}}{l^{3} \Omega^{2}}\left[2 l(5 \rho+4)-3 \epsilon(1+\rho)\left(R_{\alpha}+R_{\beta}\right)\right]\right. \\
& \left.+\frac{1}{(1+\rho)^{2}}\left[\rho^{2}(13 \rho+12)+2(1+\rho)^{2}(11 \rho+2)\right]\right\} \text {, } \\
& C_{6} \equiv \frac{c^{2}}{\epsilon \Omega^{2} l^{3}}\left\{12\left(l \epsilon-R_{\alpha}-R_{\beta}\right)+\rho\left[20 l \epsilon-12\left(R_{\alpha}+R_{\beta}\right)\right]\right\} \\
& +\frac{1}{(1+\rho)^{3}}\left[4 \rho^{3}+(1+\rho)\left(31 \rho^{2}+14 \rho+1\right)\right], \\
& C_{5} \equiv-2 \frac{c^{4}\left(R_{\alpha}+R_{\beta}\right)}{l^{5} \Omega^{4}}+\frac{c^{2}}{l^{3} \Omega^{2}(1+\rho)^{2}}\left[-4 l \epsilon(1+\rho)(5 \rho+2)+3 R_{\alpha}\left(5 \rho^{2}+12 \rho+6\right)\right. \\
& \left.+R_{\beta}\left(18 \rho^{2}+44 \rho+23\right)\right]-\frac{3 \epsilon \rho}{(1+\rho)^{3}}\left(7 \rho^{2}+6 \rho+1\right), \\
& C_{4} \equiv \frac{2 c^{2}}{\epsilon l^{3} \Omega^{2}}\left\{\left[2 l\left(R_{\beta}-2 R_{\alpha}\right)-\epsilon\left(\left(R_{\alpha}\right)^{2}+\left(R_{\beta}\right)^{2}\right)\right]\left(\frac{-c^{2}}{l^{3} \Omega^{2}}\right)+\frac{R_{\beta}}{(1+\rho)^{2}}\left[5 \rho^{2}+2 \rho+5-2 \epsilon(1+\rho)\right]\right. \\
& \left.+\frac{1}{(1+\rho)^{2}}\left[l \epsilon(1+\rho)(1+5 \rho)-6 R_{\alpha}(1+2 \rho)\right]\right\}+\frac{\rho^{2}}{(1+\rho)^{3}}(7 \rho+3) \\
& C_{3} \equiv-\frac{2 c^{4}}{l^{6}(1+\rho)^{3} \Omega^{4} \epsilon}\left[R_{\beta}\left(R_{\beta}+l \epsilon\right)+R_{\alpha}\left(4 R_{\alpha}+6 l \epsilon\right)\right]-\frac{c^{2}}{l^{3}(1+\rho)^{2} \Omega^{2} \epsilon}\{2 l \rho(1+\rho) \\
& \left.+3 R_{\alpha} \epsilon\left(5 \rho^{2}-2 \rho-1\right)+R_{\beta}\left[10(1+\rho)-\epsilon\left(3 \rho^{2}+44 \rho+18\right)\right]\right\}-\frac{\rho^{3}}{(1+\rho)^{3} \epsilon}, \\
& C_{2} \equiv \frac{c^{2}}{l^{3} \Omega^{2}}\left\{\frac{4 c^{2} R_{\alpha}}{l^{3} \Omega^{2}}\left(3 L_{\alpha}+2 l \epsilon\right)+\frac{1}{(1+\rho)^{2}}\left\{12 \epsilon R_{\alpha} \rho^{2}-8 R_{\beta}[\epsilon(1+3 \rho)-(1+\rho)]\right\}\right\}, \\
& C_{1} \equiv-\frac{c^{2} \epsilon}{l^{3} \Omega^{2}(1+\rho)^{2}}\left\{\left[2 c^{2} R_{\alpha}\left(4 R_{\alpha}+l \epsilon\right)(1+\rho)^{2}\right] \frac{\epsilon}{l^{3} \Omega^{2}}+3 \rho^{2} R_{\alpha}+2 R_{\beta}[\epsilon(1+\rho)-(1+3 \rho)]\right\} \text {, }
\end{aligned}
$$




$$
C_{0} \equiv 2\left(\frac{c^{2} R_{\alpha}}{l^{3} \Omega^{2}}\right)^{2},
$$

whereas in the other case, i.e. $s=-(r-\epsilon l)$, we end up with the algebraic equation

$$
\sum_{n=0}^{10} D_{n} \gamma^{n}=0
$$

with

$$
\begin{gathered}
D_{k}=C_{k} \quad \text { if } k=10,9,8,0, \\
D_{7} \equiv C_{7}-6 \frac{c^{2} R_{\beta}}{l^{3} \Omega^{2}}, \\
D_{6} \equiv C_{6}+24 \frac{c^{2} R_{\beta}}{l^{3} \Omega^{2} \epsilon}, \\
D_{5} \equiv C_{5}-\frac{2 c^{2} R_{\beta}}{l^{5} \Omega^{4}(1+\rho)^{2}}\left[l^{2} \Omega^{2}\left(18 \rho^{2}+38 \rho+21\right)-2 c^{2}(1+\rho)^{2}\right], \\
D_{4} \equiv C_{4}-\frac{2 c^{2} R_{\beta}}{l^{5} \Omega^{4}(1+\rho)^{2} \epsilon}\left\{4 c^{2}(1+\rho)^{2}+2 \Omega^{2} l^{2}\left[2 \epsilon(1+\rho)-\left(6 \rho^{2}+14 \rho+9\right)\right]\right\}, \\
D_{3} \equiv C_{3}+\frac{2 c^{2} R_{\beta}}{l^{3} \Omega^{2}}\left[\frac{2 c^{2}}{l^{2} \Omega^{2}}+\frac{10}{(1+\rho) \epsilon}-\frac{1}{(1+\rho)^{2}}\left(3 \rho^{2}+8 \rho+6\right)\right], \\
D_{2} \equiv C_{2}-\frac{16 c^{2} R_{\beta}}{l^{3} \Omega^{2}(1+\rho)}, \\
D_{1} \equiv C_{1}+\frac{4 c^{2} R_{\beta} \epsilon}{l^{3} \Omega^{2}(1+\rho)} .
\end{gathered}
$$

The values $R_{i}(i=1,2,3)$ of the distance of the planetoid from the Earth at the libration points $L_{1}, L_{2}, L_{3}$, respectively, obtained through the solution of Eqs. (4.38) and (4.50) are given by

$$
\begin{aligned}
& R_{1}=3.2637628817407598555 \times 10^{8} \mathrm{~m}, \\
& R_{2}=4.4892056003414800050 \times 10^{8} \mathrm{~m}, \\
& R_{3}=3.8167471569392170594 \times 10^{8} \mathrm{~m},
\end{aligned}
$$

whereas the corresponding classical Newtonian values read as [15]

$$
\begin{aligned}
& r_{1}=3.2637629578162163 \times 10^{8} \mathrm{~m}, \\
& r_{2}=4.4892055063051933 \times 10^{8} \mathrm{~m}, \\
& r_{3}=3.8167471682615924 \times 10^{8} \mathrm{~m} .
\end{aligned}
$$


By comparing these values we have

$$
\begin{gathered}
R_{1}^{(G R)}-r_{1}^{(N)}=R_{1}-r_{1}=-7.61 \mathrm{~m} \text { at } L_{1}, \\
R_{2}^{(G R)}-r_{2}^{(N)}=R_{2}-r_{2}=9.40 \mathrm{~m} \text { at } L_{2}, \\
R_{3}^{(G R)}-r_{3}^{(N)}=R_{3}-r_{3}=-1.13 \mathrm{~m} \text { at } L_{3} .
\end{gathered}
$$

Interestingly, the correction on the position of the Lagrangian point $L_{1}$ is exactly the same as the one calculated with the method described in Ref. [54], where ${ }^{3}$ the collinear solutions of the three-body problem are studied in the post-Newtonian regime. We believe that, according to the definitions involving the ratio of the distances of the planetoid from the

primaries given in Ref. [54], the equations resulting from the application of the method developed by the authors of Ref. [54] (which is the same method used in Ref. [55]) are well suited to describe only the position of $L_{1}$, and the agreement with the corrections presented here is a clue supporting our opinion.

\section{QUANTUM EFFECTS ON LAGRANGIAN POINTS}

The analysis of the previous section prepares the ground for a more appropriate definition and evaluation of quantum corrections of Lagrangian points, when the underlying classical theory of gravity is Einstein's general relativity. For this purpose, we begin by considering the analysis in Ref. [63], where the metric tensor components in a co-rotating frame for the relativistic restricted planar three-body problem in the post-Newtonian limit were obtained. With the notation of our Sec. IV, and coordinates $x^{0}=c t, x^{1}=\xi, x^{2}=\eta, x^{3}=\zeta$, the result

\footnotetext{
${ }^{3}$ As shown in Ref. [54], the general relativity corrections to $L_{1}, L_{2}$ may be of order 30 meters in the SunJupiter system. However, compared to the Earth-Moon system, a mission to test this effect at Jupiter would be exceedingly more expensive and complex to realize and could not even benefit from the use of accurate, direct laser ranging from Earth due to the large distance. The effect of the extremely harsh Jupiter radiation environment on the test spacecraft (planetoid) should also be considered to evaluate its impact on the integrity of the spacecraft and, therefore, the duration of the positioning measurements.
} 
in Ref. [63] reads as (cf. Ref. [62])

$$
\begin{aligned}
g_{00} & =1-2 \frac{R_{\alpha}}{r}-2 \frac{R_{\beta}}{s}-\frac{\Omega^{2}}{c^{2}}\left(\xi^{2}+\eta^{2}\right)+2\left[\left(\frac{R_{\alpha}}{r}\right)^{2}+\left(\frac{R_{\beta}}{s}\right)^{2}\right] \\
- & 2 \frac{\left(R_{\alpha}+R_{\beta}\right)}{l^{3}}\left(\frac{R_{\alpha}}{r}+\frac{R_{\beta}}{s}\right)\left(\xi^{2}+\eta^{2}\right)+4 \frac{R_{\alpha}}{r} \frac{R_{\beta}}{s} \\
+ & \frac{(2-\rho)}{(1+\rho)} \frac{R_{\alpha}}{r} \frac{R_{\beta}}{l}+\frac{(2 \rho-1)}{(1+\rho)} \frac{R_{\beta}}{s} \frac{R_{\alpha}}{l}-7 \frac{\xi}{l^{2}}\left(\frac{R_{\alpha}}{r} R_{\beta}-\frac{R_{\beta}}{s} R_{\alpha}\right) \\
+ & (1+\rho)^{-1} \frac{\eta^{2}}{l}\left[\rho\left(\frac{R_{\alpha}}{r}\right)^{3} \frac{R_{\beta}}{\left(R_{\alpha}\right)^{2}}+\left(\frac{R_{\beta}}{s}\right)^{3} \frac{R_{\alpha}}{\left(R_{\beta}\right)^{2}}\right], \\
\left.2 c g_{02}=-\left(1+2 \frac{R_{\alpha}}{r}+2 \frac{R_{\beta}}{s}\right) 2 \Omega \xi-8 \frac{R_{\alpha}}{r}+2 \frac{R_{\beta}}{s}\right) 2 \Omega \eta, & \left(\rho \frac{R_{\alpha}}{r}-\frac{R_{\beta}}{s}\right), \\
g_{03}=0, & -\left(1+2 \frac{R_{\alpha}}{r}+2 \frac{R_{\beta}}{s}\right) \delta_{i j}, i, j=1,2,3 .
\end{aligned}
$$

The resulting Lagrangian that describes the planetoid motion in the gravitational field of Earth and Moon reads as [64, 65]

$$
L=\frac{1}{2} \sum_{\mu, \nu=0}^{3} g_{\mu \nu} \frac{\mathrm{d} x^{\mu}}{\mathrm{d} t} \frac{\mathrm{d} x^{\nu}}{\mathrm{d} t} .
$$

We now bear in mind that, in light of second line of (4.1), the dimensionless ratio

$$
U_{\alpha}(r) \equiv \frac{R_{\alpha}}{r}=U_{\alpha}
$$

where $R_{\alpha} \equiv \frac{G \alpha}{c^{2}}$ is the gravitational radius of the primary of mass $\alpha$, gets replaced by (or mapped into)

$$
\begin{aligned}
V_{\alpha}(r) & \sim\left[1+\kappa_{2} \frac{\left(l_{P}\right)^{2}}{r^{2}}\right] U_{\alpha}(r)+\kappa_{1}\left(1+\frac{R_{m}}{R_{\alpha}}\right)\left(U_{\alpha}(r)\right)^{2}+\mathrm{O}\left(G^{3}\right) \\
& \sim\left[1+\kappa_{2} \frac{\left(l_{P}\right)^{2}}{r^{2}}\right] U_{\alpha}(r)+\kappa_{1}\left(U_{\alpha}(r)\right)^{2}
\end{aligned}
$$

because the gravitational radius $R_{m}$ of the planetoid or laser ranging test mass is indeed much smaller than $R_{\alpha}$. The same holds for the dimensionless ratio

$$
U_{\beta}(s) \equiv \frac{R_{\beta}}{s}=U_{\beta}
$$


and its effective-gravity counterpart

$$
\begin{aligned}
V_{\beta}(s) & \sim\left[1+\kappa_{2} \frac{\left(l_{P}\right)^{2}}{s^{2}}\right] U_{\beta}(s)+\kappa_{1}\left(1+\frac{R_{m}}{R_{\beta}}\right)\left(U_{\beta}(s)\right)^{2}+\mathrm{O}\left(G^{3}\right), \\
& \sim\left[1+\kappa_{2} \frac{\left(l_{P}\right)^{2}}{s^{2}}\right] U_{\beta}(s)+\kappa_{1}\left(U_{\beta}(s)\right)^{2} .
\end{aligned}
$$

By virtue of Eqs. (5.1)-(5.10), we are led to consider the effective-gravity Lagrangian

$$
\begin{aligned}
L_{V} & =\frac{c^{2}}{2}\left\{1-2\left(V_{\alpha}+V_{\beta}\right)-\frac{\Omega^{2}}{c^{2}}\left(\xi^{2}+\eta^{2}\right)+2\left[\left(V_{\alpha}\right)^{2}+\left(V_{\beta}\right)^{2}\right]\right. \\
& -2 \frac{\left(R_{\alpha}+R_{\beta}\right)}{l^{3}}\left(\xi^{2}+\eta^{2}\right)\left(V_{\alpha}+V_{\beta}\right)+4 V_{\alpha} V_{\beta} \\
& +\frac{(2-\rho)}{(1+\rho)} \frac{R_{\beta}}{l} V_{\alpha}+\frac{(2 \rho-1)}{(1+\rho)} \frac{R_{\alpha}}{l} V_{\beta}-7 \frac{\xi}{l^{2}}\left(R_{\beta} V_{\alpha}-R_{\alpha} V_{\beta}\right) \\
& \left.+(1+\rho)^{-1} \frac{\eta^{2}}{l}\left[\rho \frac{R_{\beta}}{\left(R_{\alpha}\right)^{2}}\left(V_{\alpha}\right)^{3}+\frac{R_{\alpha}}{\left(R_{\beta}\right)^{2}}\left(V_{\beta}\right)^{3}\right]\right\} \\
& -\frac{1}{2}\left(\dot{\xi}^{2}+\dot{\eta}^{2}+\dot{\zeta}^{2}\right)\left[1+2\left(V_{\alpha}+V_{\beta}\right)\right]+\Omega \eta \dot{\xi}\left[1+2\left(V_{\alpha}+V_{\beta}\right)\right] \\
& -\Omega \xi \dot{\eta}\left[1+2\left(V_{\alpha}+V_{\beta}\right)\right]-4 \frac{\Omega^{2} l}{(1+\rho)} \dot{\eta}\left(\rho V_{\alpha}-V_{\beta}\right),
\end{aligned}
$$

and the only nontrivial Euler-Lagrange equations for the planar restricted three-body problem are

$$
\frac{\mathrm{d}}{\mathrm{d} t}\left(\frac{\partial L_{V}}{\partial \dot{\xi}}\right)-\frac{\partial L_{V}}{\partial \xi}=0, \frac{\mathrm{d}}{\mathrm{d} t}\left(\frac{\partial L_{V}}{\partial \dot{\eta}}\right)-\frac{\partial L_{V}}{\partial \eta}=0 .
$$

Note that, in Refs. [13-15], we have inserted the effective-gravity map (see (5.8) and (5.10))

$$
\left(U_{\alpha}, U_{\beta}\right) \rightarrow\left(V_{\alpha}, V_{\beta}\right)
$$

in the Lagrangian of Newtonian gravity for the restricted planar three-body problem, whereas we are here inserting the same map in the Lagrangian of general relativity for the restricted three-body problem. The metric tensor with components (5.1)-(5.5) describes, within the framework of general relativity, a tiny departure from the Newtonian treatment of the restricted planar three-body problem. At that stage, one can recognize that many Newtonian-potential terms occur therein; for each of them, we apply the effective-gravity map (5.8) and (5.10) to find what we call a quantum-corrected Lagrangian.

Note however that in Ref. [66], where the authors derive quantum corrections to some known exact solutions in general relativity, they find that these metrics differ from the classical metrics only for an additional term proportional to $\left(l_{P}\right)^{2}$. Within such a framework, the running of $G$ at large $r$ has a universal character independent of masses, and there is no 
room left for $\kappa_{1}$ in the quantum-corrected Lagrangian. The two schemes are conceptually different: quantum corrections to known exact solutions of general relativity do not necessarily have the same nature as quantum corrections of metrics which represent solutions of the linearized Einstein equations and which are used in turn to derive equations of motion of interacting bodies. The insertion of the map (5.8) and (5.10) in the Lagrangian of general relativity for three bodies leads to other terms quadratic in $U_{\alpha}$ and $U_{\beta}$, which are of the same order of those already present, and hence the resulting Euler-Lagrange equations (5.12) will lead to predictions affected by $\kappa_{1}$.

To further clarify this crucial issue we point out that, if we insert the map (5.8) and (5.10) in the Lagrangian of Newtonian gravity for the restricted planar three-body problem [13], we find, with our notation, the effective potential

$$
W_{\text {eff }}=\frac{\omega^{2}}{2}\left(\xi^{2}+\eta^{2}\right)+c^{2}\left[\left(U_{\alpha}+U_{\beta}\right)+\kappa_{1}\left(\left(U_{\alpha}\right)^{2}+\left(U_{\beta}\right)^{2}\right)\right]+\mathrm{O}\left(G^{2}\right),
$$

whereas general relativity yields the effective potential (4.9), expressible in the form

$$
\begin{aligned}
W_{\mathrm{eff}} & =\frac{\Omega^{2}}{2}\left(\xi^{2}+\eta^{2}\right)+c^{2}\left[\left(U_{\alpha}+U_{\beta}\right)-\frac{1}{2}\left(\left(U_{\alpha}\right)^{2}+\left(U_{\beta}\right)^{2}\right)\right]+\mathrm{O}\left(G^{2}\right) \\
& \sim \frac{\omega^{2}}{2}\left(\xi^{2}+\eta^{2}\right)+c^{2}\left[\left(U_{\alpha}+U_{\beta}\right)-\frac{1}{2}\left(\left(U_{\alpha}\right)^{2}+\left(U_{\beta}\right)^{2}\right)\right]+\mathrm{O}\left(G^{2}\right)
\end{aligned}
$$

because $\omega^{2}=\frac{c^{2}}{l^{3}}\left(R_{\alpha}+R_{\beta}\right)=\mathrm{O}(G)$ and, by virtue of $(4.4), \Omega^{2} \sim \omega^{2}+\mathrm{O}\left(G^{2}\right)$. Hence it is possible to understand why the map (5.8) and (5.10) leads to coordinates of $L_{4}$ and $L_{5}$ in Refs. [13-15] pretty close to those of our Sec. IV.

Moreover, since the work in Refs. $[12,66]$ has studied three kinds of corrected Newtonian potential, i.e. scattering or bound states or one-particle reducible, one has to rewrite the map (5.8) and (5.10) in the form

$$
\begin{aligned}
& V_{\alpha} \sim U_{\alpha}+\left(\kappa_{1}^{\prime}+\frac{7}{2} \kappa_{0}\right)\left(U_{\alpha}\right)^{2}+\mathrm{O}\left(G^{2}\right), \\
& V_{\beta} \sim U_{\beta}+\left(\kappa_{1}^{\prime}+\frac{7}{2} \kappa_{0}\right)\left(U_{\beta}\right)^{2}+\mathrm{O}\left(G^{2}\right),
\end{aligned}
$$

which, upon defining $\kappa_{1} \equiv \kappa_{1}^{\prime}+\frac{7}{2} \kappa_{0}$, takes the form

$$
\begin{aligned}
& V_{\alpha} \sim U_{\alpha}+\kappa_{1}\left(U_{\alpha}\right)^{2}+\mathrm{O}\left(G^{2}\right), \\
& V_{\beta} \sim U_{\beta}+\kappa_{1}\left(U_{\beta}\right)^{2}+\mathrm{O}\left(G^{2}\right),
\end{aligned}
$$


where

$$
\kappa_{2} \frac{\left(l_{P}\right)^{2}}{r^{2}} U_{\alpha}=\mathrm{O}\left(G^{2}\right), \kappa_{2} \frac{\left(l_{P}\right)^{2}}{s^{2}} U_{\beta}=\mathrm{O}\left(G^{2}\right)
$$

and the parameter $\kappa_{0}$ vanishes in the scattering and one-particle reducible cases [66], whereas it equals -1 for bound states [12]. Remarkably, since $\kappa_{1}^{\prime}=3$, and $\kappa_{0}=-1$ for bound states [12], this simple calculation shows that the insertion of the map (5.15) and (5.16) into the Lagrangian of Newtonian gravity for the three-body problem leads to the effective potential

$$
W_{\mathrm{eff}}=\frac{\omega^{2}}{2}\left(\xi^{2}+\eta^{2}\right)+c^{2}\left[\left(U_{\alpha}+U_{\beta}\right)-\frac{1}{2}\left(\left(U_{\alpha}\right)^{2}+\left(U_{\beta}\right)^{2}\right)\right]+\mathrm{O}\left(G^{2}\right)
$$

which has the first three terms in common with the effective potential (5.14) of general relativity. As far as we can see, this is evidence in favour of inserting the effective-gravity map into the Lagrangian, and in favour of considering the values of $\kappa_{1}$ and $\kappa_{0}$ appropriate for bound states both below and in the analysis of Secs. II and III. Now we set to zero all time derivatives of $\xi$ and $\eta$ in Eqs. (5.12), we define the real numbers $\gamma$ and $\Gamma$ as in Sec. IV and solve numerically the resulting algebraic system for such numbers. This method leads to the following values:

\section{- noncollinear Lagrangian points:}

The planar coordinates of equilibrium points $L_{4}$ and $L_{5}$ read as

$$
\begin{gathered}
\xi_{4}=\xi_{5}=1.8752814880352634039 \times 10^{8} \mathrm{~m} \\
\eta_{4,5}= \pm 3.3290016521227759284 \times 10^{8} \mathrm{~m}
\end{gathered}
$$

which means that the differences with respect to the corresponding values provided by the Einstein theory, that plays in this scheme the role of the classical theory of reference, read as (cf. Eq. (4.33))

$$
\begin{gathered}
\xi_{4}-\xi_{4}^{(G R)}=-1.46 \mathrm{~mm} \Longrightarrow \xi_{4}-\xi_{4}^{(N)}=1.27 \mathrm{~mm}, \\
\eta_{4}-\eta_{4}^{(G R)}=-0.86 \mathrm{~mm} \Longrightarrow \eta_{4}-\eta_{4}^{(N)}=-2.45 \mathrm{~mm} .
\end{gathered}
$$

\section{- collinear Lagrangian points :}

For the libration points $L_{1}, L_{2}$ and $L_{3}$, respectively, we have found that

$$
R_{1}^{\prime}=3.2637628817345938976 \times 10^{8} \mathrm{~m}
$$




$$
\begin{aligned}
& R_{2}^{\prime}=4.4892056003375634274 \times 10^{8} \mathrm{~m} \\
& R_{3}^{\prime}=3.81674715692440418189 \times 10^{8} \mathrm{~m} .
\end{aligned}
$$

Thus, bearing in mind Eqs. (4.59)-(4.61), the differences with respect to the values expected from general relativity are

$$
\begin{aligned}
& R_{1}^{\prime}-R_{1}=-0.62 \mathrm{~mm}, \\
& R_{2}^{\prime}-R_{2}=-0.39 \mathrm{~mm}, \\
& R_{3}^{\prime}-R_{3}=-1.48 \mathrm{~mm} .
\end{aligned}
$$

Another important issue concerning both noncollinear and collinear Lagrangian points, consists in the fact that we have checked numerically that the corrections (5.22), (5.23) and (5.27)-(5.29) do not change if we set $\kappa_{2}=0$ in the Euler-Lagrange equations (5.12), because

$\kappa_{2}$ weighs the dimensionless ratios $\frac{\left(l_{P}\right)^{2}}{r^{2}}$ and $\frac{\left(l_{P}\right)^{2}}{s^{2}}$, which are extremely small at large values of $r$ and $s$.

\section{GENERAL RELATIVITY VS. EFFECTIVE GRAVITY: CONCLUDING RE- MARKS AND OPEN PROBLEMS}

The first part of this paper contributes to make more realistic the model outlined in Refs. [13-15] by considering the gravitational presence of the Sun as a perturbing effect for the Earth-Moon system. In fact we have shown that also in the quantum regime the presence of the Sun makes the planetoid ultimately escape from the triangular libration points, which therefore can be considered as "stable" equilibrium points only during the length of observations. Unless we consider solar radiation pressure, from Eqs. (2.18)-(2.20) we have obtained a plot describing the spacecraft motion about $L_{4}$ (Fig. 3) which is slightly modified if compared with the corresponding classical one (Fig. 1). If we instead take into account the solar radiation pressure (3.1), the differences between classical and quantum theory become more evident. The presence of solar radiation pressure in the classical case, in fact, makes just the planetoid go away from the Lagrangian points $L_{4}$ more rapidly (see Fig. 5), but in the quantum case, before escaping away from the libration point $L_{4}$, the planetoid is characterized by a less chaotic and irregular motion, as is clear from Fig. 6 . This feature remains true also if we consider several initial velocities for the planetoid (Figs. 
7 and 8). In particular, we have shown that the reduction of the envelope of the planetoid motion becomes more evident in the quantum case. After that, we have calculated the impulse needed for the stability of the spacecraft at $L_{4}$ both in the classical and in the quantum regime. These two values, as witnessed by Eq. (3.10), are a little bit different and therefore they suggest sending two satellites at $L_{4}$ and $L_{5}$, respectively, and checking which is the impulse truly needed for stability, in order to find out which is, between the classical and the quantum one, the best theory suited to describe these phenomena.

In the second part of the paper, we first perform a comparison between Newtonian gravity and general relativity, since of course the latter is the most successful theory describing gravitational interactions, at least in the solar system. By evaluating the points where the gradient of the potential (4.9) vanishes, we have solved the algebraic equation describing the position of Lagrangian points. The distances of noncollinear Lagrangian points from the primaries are given in terms of the solutions of Eqs. (4.14)-(4.15) (or equivalently (4.16)(4.17)). As is clear from Eq. (4.33), we have obtained corrections of the planar coordinates of the triangular libration points of the order of few millimeters and, in particular, the correction on the $\xi$-coordinate is exactly the same as the one obtained through the method outlined by the authors of Ref. [55]. As far as collinear Lagrangian points are concerned, we have to focus on Eqs. (4.38) and (4.50), from which we have evaluated corrections of the distances of the planetoid from the Earth of the order of few meters. In particular, the correction concerning $L_{1}$ is exactly the same as the one obtainable with the method of Ref. [54].

In the last part of our paper, we have outlined the features of a quantum theory whose underlying classical theory is represented by general relativity and not, as before, by Newtonian gravity $[5,12-15]$. In other words, we have dealt with a theory involving quantum corrections to Einstein gravity, rather than to Newtonian gravity. In fact, by applying the map (5.8) and (5.10) to the Lagrangian (5.6) that general relativity provides for the restricted three-body problem, we have ended up with the quantum corrected Lagrangian (5.11) which, by the means of Euler-Lagrange equations (5.12) together with the conditions $\ddot{\xi}=\ddot{\eta}=\ddot{\zeta}=\dot{\xi}=\dot{\eta}=\dot{\zeta}=\zeta=0$, has led us to the few millimeters corrections (5.22), (5.23) and (5.27)-(5.29), obtained by using the quantum coefficients $\kappa_{1}=-1 / 2$ and $\kappa_{2}=\frac{41}{10 \pi}$ of the bound-states potential discussed in Ref. [12]. We stress that, within this new scheme, quantum corrections on Newtonian quantities have been obtained through the algebraic sum 
of quantum corrections to general relativity (obtained by (5.12)) and general relativity corrections to Newton's theory (obtained by the solutions of Eqs. (4.16), (4.17), (4.38) and (4.50)). In other words, in this new approach we are no longer using the method developed in Refs. [13-15], where the map (5.8) and (5.10) was only inserted into the Newtonian Lagrangian of the restricted three-body problem, for simplicity. The possibility of mapping the effective potential of Newtonian gravity into an effective potential similar to the one of general relativity (cf. (5.19) and (5.14)) adds evidence in favor of the choice of $\kappa_{1}$ and $\kappa_{2}$ appropriate for bound states [12]. We also believe it is important to stress that we have used modern packages for dealing with all the coupled algebraic equations presented in this paper, verifying eventually that the putative solution does satisfy the original set.

In conclusion, as far as we can see, the implications of calculations presented here and in our previous work are as follows.

\section{A. Noncollinear Lagrangian points}

(i) Quantum corrections to Newtonian planar coordinates of $L_{4}$ and $L_{5}$ and to the corresponding general relativity values are $(1.27 \mathrm{~mm},-2.45 \mathrm{~mm})$ and $(-1.46 \mathrm{~mm}, 0.86 \mathrm{~mm})$, respectively. If it were possible to obtain an experimental verification of this prediction, it might provide encouraging (but not conclusive) evidence in favor of effective theories of gravity with values $\kappa_{1}=-1 / 2$ and $\kappa_{2}=41 /(10 \pi)$ of the bound-states potential.

(ii) If the departure from the equilateral triangle picture of Newtonian theory is instead close to $(2.73 \mathrm{~mm},-1.59 \mathrm{~mm})$ for the planar coordinates of $L_{4}$ and $L_{5}$, this may provide another nontrivial test of classical general relativity.

\section{B. Collinear Lagrangian points}

(iii) If the differences with respect to the classical Newtonian distances of the planetoid at $L_{1}, L_{2}$ and $L_{3}$ from the Earth were of order $(-7.61 \mathrm{~m}, 9.40 \mathrm{~m},-1.13 \mathrm{~m})$, we would have to consider this fact as another confirmation of general relativity. Here we note that the deviation of $L_{2}$ from the Newtonian prediction should be taken into account in the design of science and/or exploration missions conceived to station at $L_{2}$ with an accuracy around 
TABLE I: Distances $r_{i}$ from the Earth and planar coordinates $\left(\xi_{i}, \eta_{i}\right)$ of the planetoid at all Lagrangian points $L_{i}$ in the classical Newtonian theory, General Relativity and the quantum regime, the latter being obtained through the Lagrangian $L_{V}$ (5.11) and with the coefficients of the bound-states potential $\kappa_{1}=-1 / 2$ and $\kappa_{2}=41 /(10 \pi)$ taken from Ref.

$[12]$.

\begin{tabular}{|c|l|l|l|}
\hline$L_{i}$ & Newtonian Gravity & General Relativity & Quantum regime \\
\hline \multirow{5}{*}{$L_{1}$} & $r_{1}=3.263762957816216 \times 10^{8} \mathrm{~m}$ & $r_{1}=3.263762881740760 \times 10^{8} \mathrm{~m}$ & $r_{1}=3.263762881734594 \times 10^{8} \mathrm{~m}$ \\
\cline { 2 - 5 } & $\xi_{1}=3.217044465246977 \times 10^{8} \mathrm{~m}$ & $\xi_{1}=3.217044369763247 \times 10^{8} \mathrm{~m}$ & $\xi_{1}=3.217044369757081 \times 10^{8} \mathrm{~m}$ \\
& $\eta_{1}=0$ & $\eta_{1}=0$ & $\eta_{1}=0$ \\
\hline \multirow{3}{*}{$L_{2}$} & $r_{2}=4.489205506305193 \times 10^{8} \mathrm{~m}$ & $r_{2}=4.489205600341480 \times 10^{8} \mathrm{~m}$ & $r_{2}=4.489205600337563 \times 10^{8} \mathrm{~m}$ \\
\cline { 2 - 5 } & $\xi_{2}=4.442487013735948 \times 10^{8} \mathrm{~m}$ & $\xi_{2}=4.442487088363968 \times 10^{8} \mathrm{~m}$ & $\xi_{2}=4.442487088360051 \times 10^{8} \mathrm{~m}$ \\
& $\eta_{2}=0$ & $\eta_{2}=0$ & $\eta_{2}=0$ \\
\hline \multirow{3}{*}{$L_{3}$} & $r_{3}=3.816747168261592 \times 10^{8} \mathrm{~m}$ & $r_{3}=3.816747156939217 \times 10^{8} \mathrm{~m}$ & $r_{3}=3.816747156924404 \times 10^{8} \mathrm{~m}$ \\
\cline { 2 - 5 } & $\xi_{3}=-3.863465660830824 \times 10^{8} \mathrm{~m}$ & $\xi_{3}=-3.863465668916729 \times 10^{8} \mathrm{~m}$ & $\xi_{3}=-3.863465668901917 \times 10^{8} \mathrm{~m}$ \\
& $\eta_{3}=0$ & $\eta_{3}=0$ & $\eta_{3}=0$ \\
\hline \multirow{3}{*}{$L_{4}$} & $r_{4}=3.843999990295860 \times 10^{8} \mathrm{~m}$ & $r_{4}=3.843999999999861 \times 10^{8} \mathrm{~m}$ & $r_{4}=3.843999999985078 \times 10^{8} \mathrm{~m}$ \\
\cline { 2 - 5 } & $\xi_{4}=1.875281488022487 \times 10^{8} \mathrm{~m}$ & $\xi_{4}=1.875281488049864 \times 10^{8} \mathrm{~m}$ & $\xi_{4}=1.875281488035263 \times 10^{8} \mathrm{~m}$ \\
& $\eta_{4}=3.329001652147382 \times 10^{8} \mathrm{~m}$ & $\eta_{4}=3.329001652131416 \times 10^{8} \mathrm{~m}$ & $\eta_{4}=3.329001652122776 \times 10^{8} \mathrm{~m}$ \\
\hline \multirow{3}{*}{$L_{5}$} & $r_{5}=3.843999990295860 \times 10^{8} \mathrm{~m}$ & $r_{5}=3.843999999999861 \times 10^{8} \mathrm{~m}$ & $r_{5}=3.843999999985078 \times 10^{8} \mathrm{~m}$ \\
\cline { 2 - 5 } & $\xi_{5}=1.875281488022487 \times 10^{8} \mathrm{~m}$ & $\xi_{5}=1.875281488049864 \times 10^{8} \mathrm{~m}$ & $\xi_{5}=1.875281488035263 \times 10^{8} \mathrm{~m}$ \\
& $\eta_{5}=-3.329001652147382 \times 10^{8} \mathrm{~m}$ & $\eta_{5}=-3.329001652131416 \times 10^{8} \mathrm{~m}$ & $\eta_{5}=-3.329001652122776 \times 10^{8} \mathrm{~m}$ \\
\hline
\end{tabular}

10 meters or less.

(iv) It is remarkable that, with the values of $\kappa_{1}$ and $\kappa_{2}$ characterizing the bound-state potential, the quantum corrections on the classical Newtonian distances from the Earth are (-7.61 m, $9.40 \mathrm{~m},-1.13 \mathrm{~m})$, which coincide with those we have obtained within the general relativity context up to millimeter effects that cannot be easily detected, because they are only a tiny fraction of the correction to Newtonian values. This fact may be interpreted as a clue which shows how the quantum values $\kappa_{1}=-\frac{1}{2}, \kappa_{2}=\frac{41}{10 \pi}$ of the bound-states potential of Ref. [12] are intimately connected with the Einstein theory.

(v) In the case in which the quantum theory is ruled by the Lagrangian (5.11), the quantum corrections to general relativity become $(-0.62 \mathrm{~mm},-0.39 \mathrm{~mm},-1.48 \mathrm{~mm})$ at $L_{1}, L_{2}, L_{3}$, respectively.

A summary of all quantities involved in this paper along with all corrections discussed above is reported for clarity in Tabs. I and II. As far as we can see, our detailed calculations 
TABLE II: Corrections to the distances of $L_{1}, L_{2}$ and $L_{3}$ from the Earth and to the planar coordinates of $L_{4}$ and $L_{5}$. In the first column we have the general relativity corrections to Newtonian theory as obtained from Eqs. (4.38), (4.50), (4.16) and (4.17), respectively. The second column shows quantum corrections to general relativity given by the Euler-Lagrange equations involving the Lagrangian $L_{V}$ (5.11) and the quantum coefficients of the bound-states potential. The last column displays quantum corrections to Newtonian values calculated as the algebraic sum of the corresponding quantities in the two previous columns.

\begin{tabular}{|l|l|l|l|}
\hline$L_{i}$ & General Relativity-Newton & Quantum-General Relativity & Quantum-Newton \\
\hline$L_{1}$ & $-7.61 \mathrm{~m}$ & $-0.62 \mathrm{~mm}$ & $-7.61 \mathrm{~m}$ \\
\hline$L_{2}$ & $9.40 \mathrm{~m}$ & $-0.39 \mathrm{~mm}$ & $9.40 \mathrm{~m}$ \\
\hline$L_{3}$ & $-1.13 \mathrm{~m}$ & $-1.48 \mathrm{~mm}$ & $-1.13 \mathrm{~m}$ \\
\hline$L_{4}$ & $(2.73 \mathrm{~mm},-1.59 \mathrm{~mm})$ & $(-1.46 \mathrm{~mm},-0.86 \mathrm{~mm})$ & $(1.27 \mathrm{~mm},-2.45 \mathrm{~mm})$ \\
\hline$L_{5}$ & $(2.73 \mathrm{~mm},-1.59 \mathrm{~mm})$ & $(-1.46 \mathrm{~mm},-0.86 \mathrm{~mm})$ & $(1.27 \mathrm{~mm},-2.45 \mathrm{~mm})$ \\
\hline
\end{tabular}

show clearly that the measurement we are proposing in the Earth-Moon system represents a new testbed for general relativity and effective field theories of gravity. In fact, given the large value of the deviation of $L_{1}$ from the Newtonian prediction, it is possible to study a new laser ranging test of general relativity to measure the 7.61-meter correction to the $L_{1}$ Lagrangian point, an observable never used before in the Sun-Earth-Moon system. Performing such an experiment requires controlling the propulsion to precisely reach $L_{1}$ (also with the help of laser ranging), an instrumental accuracy comparable to the measurement of the lunar geodesic precession or better (achievable with a suitably-designed laser-ranging test mass under study), understanding systematic effects resulting from thermal radiation and multibody gravitational perturbations. To assess the feasibility of this experiment requires a dedicated study and R\&D activity. The result of this work will then be the basis to consider a second-generation experiment to study deviations of effective field theories of gravity from general relativity in the Sun-Earth-Moon system. 


\section{Acknowledgments}

G. E. is grateful to Dipartimento di Fisica of Federico II University for hospitality and

support. The work of S. D. has been supported by the INFN funding of the MoonLIGHT-2 experiment. The authors are grateful to V. Brumberg, J. F. Donoghue, M. Efroimsky, S. Kopeikin for correspondence, to V. Tiouchov for help in translating section 8.4 of Ref. [63], and to E. Calloni for encouragement.

\section{APPENDIX A: EVALUATION OF THE GRADIENT OF $w$ IN GENERAL REL-}

\section{ATIVITY}

By virtue of formulas (4.7) and (4.8), one finds

$$
\begin{gathered}
\frac{\partial}{\partial \xi}\left(r^{-p}\right)=-p r^{-p-2}\left(\xi+\frac{\rho l}{(1+\rho)}\right) \\
\frac{\partial}{\partial \xi}\left(s^{-p}\right)=-p s^{-p-2}\left(\xi-\frac{l}{(1+\rho)}\right) \\
\frac{\partial}{\partial \eta}\left(r^{-p}\right)=-p r^{-p-2} \\
\frac{\partial}{\partial \eta}\left(s^{-p}\right)=-p s^{-p-2}
\end{gathered}
$$

that we have computed with $p=1,2,3$ to obtain Eqs. (4.12) and (4.13) for the components of $\operatorname{grad}(w)$, where the functions $w_{1} \ldots w_{4}$ are defined by

$$
\begin{aligned}
W_{1}(\xi, \eta, r) & \equiv \xi \Omega^{2}+\frac{\Omega^{4} \xi\left(\xi^{2}+\eta^{2}\right)}{2 c^{2}}+3 \Omega^{2} \xi \frac{R_{\alpha}}{r}+\frac{7}{2} \frac{R_{\beta} l \Omega^{2}}{(1+\rho)} \frac{1}{r} \\
& +\left(\xi+\frac{\rho l}{(1+\rho)}\right) \frac{1}{r^{3}}\left\{c^{2} R_{\alpha}\left(\frac{R_{\alpha}}{r}-1\right)+\Omega^{2}\left[-\frac{3}{2} R_{\alpha}\left(\xi^{2}+\eta^{2}\right)\right.\right. \\
& \left.\left.-\frac{7}{2} \frac{R_{\beta} l \xi}{(1+\rho)}+\frac{3}{2} \frac{\rho}{(1+\rho)^{2}} \frac{R_{\beta} l^{2} \eta^{2}}{r^{2}}+\frac{(2-\rho)}{2(1+\rho)^{2}} R_{\beta} l^{2}\right]\right\} \\
W_{2}(\xi, \eta, s) & \equiv 3 \Omega^{2} \xi \frac{R_{\beta}}{s}-\frac{7}{2} \frac{R_{\beta} l \Omega^{2}}{(1+\rho)} \frac{1}{s} \\
& +\left(\xi-\frac{l}{(1+\rho)}\right) \frac{1}{s^{3}}\left\{c^{2} R_{\beta}\left(\frac{R_{\beta}}{s}-1\right)+\Omega^{2}\left[-\frac{3}{2} R_{\beta}\left(\xi^{2}+\eta^{2}\right)\right.\right. \\
& \left.\left.+\frac{7}{2} \frac{R_{\beta} l \xi}{(1+\rho)}+\frac{3}{2} \frac{1}{(1+\rho)^{2}} \frac{R_{\beta} l^{2} \eta^{2}}{s^{2}}+\frac{(2 \rho-1)}{2(1+\rho)^{2}} R_{\beta} l^{2}\right]\right\},
\end{aligned}
$$




$$
\begin{gathered}
W_{3}(\xi, \eta, r) \equiv \Omega^{2}+\frac{\Omega^{4}}{2 c^{2}}\left(\xi^{2}+\eta^{2}\right)+\frac{c^{2} R_{\alpha}}{r^{3}}\left(\frac{R_{\alpha}}{r}-1\right)+3 \Omega^{2} \frac{R_{\alpha}}{r} \\
-\frac{3}{2} \Omega^{2}\left(\xi^{2}+\eta^{2}\right) \frac{R_{\alpha}}{r^{3}}-\frac{7}{2} \frac{R_{\beta} l \xi \Omega^{2}}{(1+\rho)} \frac{1}{r^{3}} \\
+\frac{R_{\beta} l^{2} \Omega^{2}}{2(1+\rho)^{2}} \frac{\rho}{r^{3}}\left(3 \frac{\eta^{2}}{r^{2}}-2\right)+\frac{R_{\beta} l^{2} \Omega^{2}}{2(1+\rho)^{2}} \frac{(2-\rho)}{r^{3}}, \\
W_{4}(\xi, \eta, s) \equiv \frac{c^{2} R_{\beta}}{s^{3}}\left(\frac{R_{\beta}}{s}-1\right)+3 \Omega^{2} \frac{R_{\beta}}{s}-\frac{3}{2} \Omega^{2}\left(\xi^{2}+\eta^{2}\right) \frac{R_{\beta}}{s^{3}}+\frac{7}{2} \frac{R_{\beta} l \xi \Omega^{2}}{(1+\rho)} \frac{1}{s^{3}} \\
+\frac{R_{\beta} l^{2} \Omega^{2}}{2(1+\rho)^{2}} \frac{1}{s^{3}}\left(3 \frac{\eta^{2}}{s^{2}}-2\right)+\frac{R_{\beta} l^{2} \Omega^{2}}{2(1+\rho)^{2}} \frac{(2 \rho-1)}{s^{3}} .
\end{gathered}
$$

[1] List of Jupiter Trojans, Minor Planet Center, 25 February 2014.

[2] R. A. Freitas and F. Valdes, A search for natural or artificial objects located at the Earth-Moon libration points, Icarus 42, 442 (1980).

[3] M. Connors, P. Wiegert, and C. Veillet, Earth's Trojan asteroid, Nature (London) 475, 481 (2011).

[4] F. Zegler and B. Kutter, AIAA Space 2010 Conference \& Exposition.

[5] J. F. Donoghue, Leading quantum correction to the Newtonian potential, Phys. Rev. Lett. 72, 2996 (1994).

[6] J. F. Donoghue, General relativity as an effective field theory: the leading quantum corrections, Phys. Rev. D 50, 3874 (1994).

[7] J. F. Donoghue, The effective field theory treatment of quantum gravity, AIP Conf. Proc. 1483, 73 (2012).

[8] I. J. Muzinich and S. Vokos, Long range forces in quantum gravity, Phys. Rev. D 52, 3472 (1995).

[9] H. W. Hamber and S. Liu, On the quantum corrections to the Newtonian potential, Phys. Lett. B 357, 51 (1995).

[10] A. A. Akhundov, S. Bellucci, and A. Shiekh, Gravitational interaction to one loop in effective quantum gravity, Phys. Lett. B 395, 16 (1997).

[11] I. B. Khriplovich and G. G. Kirilin, Quantum power correction to the Newton law, Sov. Phys. JETP 95, 981 (2002). 
[12] N. E. J. Bjerrum-Bohr, J. F. Donoghue, and B. R. Holstein, Quantum gravitational corrections to the nonrelativistic scattering potential of two masses, Phys. Rev. D 67, 084033 (2003).

[13] E. Battista and G. Esposito, Restricted three-body problem in effective-field-theory models of gravity, Phys. Rev. D 89, 084030 (2014).

[14] E. Battista and G. Esposito, Full three-body problem in effective-field-theory models of gravity, Phys. Rev. D 90, 084010 (2014).

[15] E. Battista, S. Dell' Agnello, G. Esposito and J. Simo, Quantum effects on Lagrangian points and displaced periodic orbits in the Earth-Moon system, Phys. Rev. D 91, 084041 (2015).

[16] H. Poincaré, The three-body problem and the equations of dynamics, Acta Mathematica 13, 1 (1890); On the three-body problem, Bull. Astronomique 8, 12 (1891).

[17] H. Poincaré, Les Methodes Nouvelles de la Mecanique Celeste (Gauthier-Villars, Paris, 1892), reprinted as New Methods of Celestial Mechanics, edited by D. L. Goroff (American Institute of Physics, College Park, 1993).

[18] L. A. Pars, A Treatise on Analytical Dynamics (Heinemann, London, 1965).

[19] Z. Altamimi, X. Collilieux, J. Legrand et al., ITRF2005: A new release of the international terrestrial reference frame based on time series of station positions and earth orientation parameters, J. Geophys. Res. 112, B09401 (2007).

[20] M. R. Pearlman, J. J. Degnan, and J. M. Bosworth, The international laser ranging service, Adv. Space Res. 30, 135 (2002).

[21] P. L. Bender et al., The lunar experiment, Science 182, 229 (1973).

[22] J. G. Williams, S. G. Turyshev, and D. H. Boggs, Progress in lunar laser ranging tests of relativistic gravity, Phys. Rev. Lett. 93, 261101 (2004).

[23] I. I. Shapiro, R. D. Reasenberg, J. F. Chandler, and R. W. Babcock, Measurement of the de Sitter precession of the moon: A relativistic three-body effect, Phys. Rev. Lett. 61, 2643 (1988).

[24] S. Dell'Agnello et al., Probing general relativity and new physics with lunar laser ranging, Nucl. Instr. Methods Phys. Res. A 692, 275 (2012).

[25] M. Martini, S. Dell'Agnello et al., MoonLIGHT: A USA-Italy lunar laser ranging retroreflector array for the 21st century, Planet. Space Sci. 74, 276 (2012).

[26] R. March, G. Bellettini, R. Tauraso, and S. Dell'Agnello, Constraining spacetime torsion with the Moon and Mercury, Phys. Rev. D 83, 104008 (2011). 
[27] R. March, G. Bellettini, R. Tauraso, and S. Dell'Agnello, Constraining spacetime torsion with LAGEOS, Gen. Relativ. Gravit. 43, 3099 (2011).

[28] S. Dell'Agnello et al., Creation of the new industry-standard space test of laser retroreflectors for the GNSS and LAGEOS, J. Adv. Space Res. 47, 822 (2011).

[29] S. Dell'Agnello et al., Next-generation laser retroreflectors for GNSS, solar system exploration, geodesy, gravitational physics and earth-observation, in ESA Proc. Int. Conf. on Space Optics (Tenerife, Spain, Oct. 2014).

[30] D. Currie, S. Dell'Agnello, G. O. Delle Monache, B. Behr, and J. G. Williams, A lunar laser ranging retroreflector array for the 21st century, Nucl. Phys. B Proc. Suppl. 243, 218 (2013).

[31] S. Dell'Agnello et al., Fundamental physics and absolute positioning metrology with the MAGIA lunar orbiter, Exp. Astron. 32, 19 (2011).

[32] D. Vokrouhlicky, A note on the solar radiation perturbations of lunar motion, Icarus 126, 293 (1997).

[33] J. Polchinski, String Theory (Cambridge University Press, Cambridge, 1998).

[34] C. Rovelli, Quantum Gravity (Cambridge University Press, Cambridge, 2004).

[35] R. Penrose and M. A. H. MacCallum, Twistor theory: an approach to the quantization of fields and spacetime, Phys. Rep. 6 C, 241 (1973).

[36] G. Esposito, An introduction to quantum gravity, UNESCO Encyclopedia (UNESCO, Paris, France, 2011).

[37] B. S. DeWitt, Quantum theory of gravity. III. Applications of the covariant theory, Phys. Rev. 162, 1239 (1967).

[38] C. Kiefer and M. Krämer, Quantum gravitational contributions to the CMB anisotropy spectrum, Phys. Rev. Lett. 108, 021301 (2012).

[39] D. Bini, G. Esposito, K. Kiefer, M. Krämer, and F. Pessina, On the modification of the cosmic microwave background anisotropy spectrum from canonical quantum gravity, Phys. Rev. D 87, 104008 (2013).

[40] D. Bini and G. Esposito, Perturbative evaluation of the scalar two-point function in the cosmic microwave background power spectrum, Phys. Rev. D 89, 084032 (2014).

[41] A. Yu. Kamenshchik, A. Tronconi, and G. Venturi, Inflation and quantum gravity in a BornOppenheimer context, Phys. Lett. B 726, 518 (2013).

[42] A. Yu. Kamenshchik, A. Tronconi, and G. Venturi, Signatures of quantum gravity in a Born- 
Oppenheimer context, Phys. Lett. B 734, 72 (2014).

[43] A. Ashoorioon, P. S. Bhupal Dev, and A. Mazumdar, Implications of purely classical gravity for inflationary tensor modes, Mod. Phys. Lett. A 29, 1450163 (2914); L. Krauss and F. Wilczek, Using cosmology to establish the quantization of gravity, Phys. Rev. D 89, 047501 (2014).

[44] J. Simo and C. R. McInnes, Solar sail trajectories at the earth-moon Lagrange points, in 59th International Astronomical Congress, Glasgow, Scotland, 2008.

[45] J. Simo and C. R. McInnes, Displaced periodic orbits with low-thrust propulsion in the earthmoon system, in 19th AAS/AIAA Space Flight Mechanics Meeting, Savannah, Georgia, 2009.

[46] J. Simo and C. R. McInnes, Solar sail orbits at the earth-moon libration points, Comm. Nonlinear Sci. Numer. Simulat. 14, 4191 (2009).

[47] J. Simo and C. R. McInnes, Asymptotic analysis of displaced lunar orbits, J. of Guidance, Control, and Dynamics 32, 1666 (2009).

[48] J. Simo and C. R. McInnes, Displaced solar sail orbits: dynamics and applications, in 20th AAS/AIAA Space Flight Mechanics Meeting, San Diego, California, 2010.

[49] J. Simo and C. R. McInnes, Designing displaced lunar orbits using low-thrust propulsion, J. of Guidance, Control, and Dynamics 33, 259 (2010).

[50] J. Simo and C. R. McInnes, Feedback stabilization of displaced periodic orbits: application to binary asteroids, Acta Astronautica 96, 106 (2014).

[51] C. R. McInnes, Solar Sailing: Technology, Dynamics and Mission Applications (Springer Praxis, London, 1999).

[52] B. D. Tapley and J. M. Lewallen, Solar influence on satellite motion near the stable earth-moon libration points, AIAA J. 2, 728 (1964).

[53] H. Asada, Gravitational wave forms for a three-body system in Lagrange's orbit: Parameter determinations and a binary source test, Phys. Rev. D 80, 064021 (2009).

[54] K. Yamada and H. Asada, Collinear solution to the general relativistic three-body problem, Phys. Rev. D 82, 104019 (2010).

[55] K. Yamada and H. Asada, Triangular solution to the general relativistic three-body problem for general masses, Phys. Rev. D 86, 124029 (2012).

[56] K. Yamada and H. Asada, Post-Newtonian effects on the stability of the triangular solution in the three-body problem for general masses, arXiv:1505.04534v1 (2015). 
[57] V. Szebehely, Theory of Orbits: the Restricted Problem of Three Bodies (Academic Press, New York, 1967).

[58] T. Levi-Civita, The N-Body Problem in General Relativity (Reidel, Dordrecht, 1941).

[59] K. B. Bhatnagar and P. P. Hallan, Existence and stability of $L_{4,5}$ in the relativistic restricted three-body problem, Cel. Mech. 69, 271 (1998).

[60] C. N. Douskos and E. A. Perdios, On the stability of equilibrium points in the relativistic restricted three-body problem, Cel. Mech. 82, 317 (2002).

[61] V. A. Brumberg, Special solutions in a simplified restricted three-body problem with gravitational radiation taken into account, Cel. Mech. 85, 269 (2003).

[62] E. Krefetz, Restricted three-body problem in the post-Newtonian approximation, Astron. J. 72, 471 (1967).

[63] V. A. Brumberg, Relativistic Celestial Mechanics (Nauka, Moscow, 1972).

[64] L. F. Wanex, Chaotic amplification in the relativistic restricted three-body problem, Z. Naturforsch. 58a, 13 (2003).

[65] J. Foster and J. D. Nightingale, A Short Course in General Relativity (Springer, New York, 1995).

[66] N. E. J. Bjerrum-Bohr, J. F. Donoghue, and B. R. Holstein, Quantum corrections to the Schwarzschild and Kerr metrics, Phys. Rev. D 68, 084005 (2003). 